\title{
Constraints on black-hole charges with the 2017 EHT observations of M87*
}

Prashant Kocherlakota $\odot,{ }_{1}^{1}$ Luciano Rezzolla, ${ }^{1-3}$ Heino Falcke, ${ }^{4}$ Christian M. Fromm, ${ }^{5,6,1}$ Michael Kramer, ${ }^{7}$ Yosuke Mizuno, ${ }^{8,9}$ Antonios Nathanail ${ }^{9,10}$ Héctor Olivares, ${ }^{4}$ Ziri Younsi ${ }^{11,9}$ Kazunori Akiyama, ${ }^{12,13,5}$ Antxon Alberdi, ${ }^{14}$ Walter Alef, ${ }^{7}$ Juan Carlos Algaba, ${ }^{15}$ Richard Anantua, ${ }^{5,6,16}$ Keiichi Asada ${ }^{17}$ Rebecca Azulay ${ }_{18,19,7}$ Anne-Kathrin Baczko, ${ }^{7}$ David Ball, ${ }^{20}$ Mislav Baloković, ${ }^{5,6}$ John Barrett, ${ }^{12}$ Bradford A. Benson, ${ }^{21,22}$ Dan Bintley, ${ }^{23}$ Lindy Blackburn, ${ }^{5,6}$ Raymond Blundell, ${ }^{6}$ Wilfred Boland, ${ }^{24}$ Katherine L. Bouman, ${ }^{5,6,25}$ Geoffrey C. Bower, ${ }^{26}$ Hope Boyce, ${ }^{27,28}$ Michael Bremer, ${ }^{29}$ Christiaan D. Brinkerink, ${ }^{4}$ Roger Brissenden, ${ }^{5,6}$ Silke Britzen, ${ }^{7}$ Avery E. Broderick, ${ }^{30-32}$ Dominique Broguiere, ${ }^{29}$ Thomas Bronzwaer, ${ }^{4}$ Do-Young Byun, ${ }^{33,34}$ John E. Carlstrom, ${ }^{35,22,36,37}$ Andrew Chael, ${ }^{38,39}$ Chi-kwan Chan ${ }^{20,40}$ Shami Chatterjee, ${ }^{41}$ Koushik Chatterjee, ${ }^{42}$ Ming-Tang Chen, ${ }^{26}$ Yongjun Chen (陈永军) ${ }^{43,44}$ Paul M. Chesler, ${ }^{5}$ Ilje Cho, ${ }^{33,34}$ Pierre Christian, ${ }^{45}$ John E. Conway, ${ }^{46}$ James M. Cordes, ${ }^{41}$ Thomas M. Crawford, ${ }^{22,35}$ Geoffrey B. Crew ${ }^{12}$ Alejandro Cruz-Osorio, ${ }^{9}$ Yuzhu Cui, ${ }^{47,48}$ Jordy Davelaar, ${ }^{49,16,4}$ Mariafelicia De Laurentis, $, 0,9,51$

Roger Deane, ${ }^{52-54}$ Jessica Dempsey, ${ }^{23}$ Gregory Desvignes, ${ }^{55}$ Sheperd S. Doeleman, ${ }^{5,6}$ Ralph P. Eatough, ${ }^{56,7}$

Joseph Farah, ${ }^{6,5,57}$ Vincent L. Fish, ${ }^{12}$ Ed Fomalont, ${ }^{58}$ Raquel Fraga-Encinas, ${ }^{4}$ Per Friberg, ${ }^{23}$ H. Alyson Ford, ${ }^{59}$ Antonio Fuentes, ${ }^{14}$ Peter Galison, ${ }^{5,60,61}$ Charles F. Gammie ${ }^{62,63}$ Roberto García, ${ }^{29}$ Olivier Gentaz, ${ }^{29}$ Boris Georgiev, ${ }^{31,32}$

Ciriaco Goddi, ${ }^{4,64}$ Roman Gold, ${ }^{65,30}$ José L. Gómez, ${ }^{14}$ Arturo I. Gómez-Ruiz, ${ }^{66,67}$ Minfeng Gu (顾敏峰), ${ }^{4,68}$ Mark Gurwell, ${ }^{6}$ Kazuhiro Hada ${ }^{47,48}$ Daryl Haggard, ${ }^{27,28}$ Michael H. Hecht, ${ }^{12}$ Ronald Hesper ${ }^{69}$ Luis C. Ho (何子山) ${ }^{70,71}$ Paul Ho, ${ }^{17}$ Mareki Honma, ${ }^{47,48,72}$ Chih-Wei L. Huang, ${ }^{17}$ Lei Huang (黄磊) ${ }^{43,68}$ David H. Hughes, ${ }^{66}$ Shiro Ikeda, ${ }^{13,73-75}$ Makoto Inoue, ${ }^{17}$ Sara Issaoun, ${ }^{4}$ David J. James, ${ }^{5,6}$ Buell T. Jannuzi, ${ }^{20}$ Michael Janssen, ${ }^{7}$ Britton Jeter, ${ }^{31,32}$ Wu Jiang (江悟), ${ }^{43}$ Alejandra Jimenez-Rosales, ${ }^{4}$ Michael D. Johnson, ${ }^{5,6}$ Svetlana Jorstad, ${ }^{76,77}$ Taehyun Jung, ${ }^{33,34}$ Mansour Karami, ${ }^{30,31}$ Ramesh Karuppusamy, ${ }^{7}$ Tomohisa Kawashima, ${ }^{78}$ Garrett K. Keating, ${ }^{6}$ Mark Kettenis, ${ }^{79}$ Dong-Jin Kim, ${ }^{7}$ Jae-Young Kim, ${ }^{33,7}$ Jongsoo Kim, ${ }^{33}$ Junhan Kim, ${ }^{20,25}$ Motoki Kino, ${ }^{13,80}$ Jun Yi Koay, ${ }^{17}$ Yutaro Kofuji, ${ }^{47,72}$ Patrick M. Koch, ${ }^{17}$ Shoko Koyama, ${ }_{17}$ Carsten Kramer, ${ }^{29}$ Thomas P. Krichbaum, ${ }^{7}$ Cheng-Yu Kuo, ${ }^{81,17}$ Tod R. Lauer, ${ }^{82}$ Sang-Sung Lee, ${ }^{33}$ Aviad Levis, ${ }^{25}$ Yan-Rong Li (李彦荣), ${ }^{83}$ Zhiyuan Li (李志远) ${ }^{84,85}$ Michael Lindqvist, ${ }^{46}$ Rocco Lico, ${ }^{14,7}$ Greg Lindahl, ${ }^{6}$ Jun Liu (刘俊), ${ }^{7}$ Kuo Liu, ${ }^{7}$ Elisabetta Liuzzo, ${ }^{86}$ Wen-Ping Lo, ${ }^{17,87}$ Andrei P. Lobanov, Laurent Loinard, ${ }^{8,89}$ Colin Lonsdale, ${ }^{12}$ Ru-Sen Lu (路如森), ${ }^{43,44,7}$ Nicholas R. MacDonald, Jirong Mao (毛基荣), ${ }^{70-92}$ Nicola Marchili, ${ }^{86,7}$ Sera Markoff, ${ }^{42,93}$ Daniel P. Marrone, ${ }^{20}$ Alan P. Marscher, ${ }^{76}$ Iván Martí-Vidal, ${ }^{18,19}$ Satoki Matsushita, ${ }^{17}$

Lynn D. Matthews, ${ }^{12}$ Lia Medeiros, ${ }^{94,20}$ Karl M. Menten, ${ }^{7}$ Izumi Mizuno, ${ }^{23}$ James M. Moran ${ }^{5,6}$ Kotaro Moriyama, ${ }^{12,47}$ Monika Moscibrodzka, ${ }^{4}$ Cornelia Müller, ${ }_{95}^{7,4}$ Gibwa Musoke, ${ }^{42,4}$ Alejandro Mus Mejías, ${ }^{18,19}$ Hiroshi Nagai, ${ }^{13,48}$

Neil M. Nagar, ${ }^{95}$ Masanori Nakamura, ${ }^{96,17}$ Ramesh Narayan, ${ }^{5,6}$ Gopal Narayanan, ${ }^{97}$ Iniyan Natarajan, ${ }^{54,52,98}$ Joseph Neilsen, ${ }^{99}$ Roberto Neri, ${ }^{29}$ Chunchong Ni, ${ }^{31,32}$ Aristeidis Noutsos, ${ }^{7}$ Michael A. Nowak, ${ }^{100}$ Hiroki Okino, ${ }^{47,72}$ Gisela N. Ortiz-León, ${ }^{7}$ Tomoaki Oyama, ${ }^{47}$ Feryal Özel, ${ }^{20}$ Daniel C. M. Palumbo, ${ }^{5,6}$ Jongho Park, ${ }^{17}$ Nimesh Patel, ${ }^{6}$

Ue-Li Pen ${ }^{30,101-103}$ Dominic W. Pesce, ${ }^{5,6}$ Vincent Piétu, ${ }^{29}$ Richard Plambeck, ${ }^{104}$ Aleksandar PopStefanija, ${ }^{97}$

Oliver Porth, ${ }^{42,9}$ Felix M. Pötzl, ${ }^{7}$ Ben Prather, ${ }^{62}$ Jorge A. Preciado-López, ${ }^{30}$ Dimitrios Psaltis, ${ }^{20}$ Hung-Yi Pu, ${ }^{105,17,30}$ Venkatessh Ramakrishnan, ${ }^{95}$ Ramprasad Rao, ${ }^{26}$ Mark G. Rawlings, ${ }^{23}$ Alexander W. Raymond, ${ }^{5,6}$ Angelo Ricarte, ${ }^{5,6}$ Bart Ripperda, ${ }^{106,16}$ Freek Roelofs, ${ }^{4}$ Alan Rogers ${ }^{12}$ Eduardo Ros, ${ }^{7}$ Mel Rose, ${ }^{20}$ Arash Roshanineshat, ${ }^{20}$ Helge Rottmann, ${ }^{7}$ Alan L. Roy, ${ }^{7}$ Chet Ruszczyk, ${ }^{12}$ Kazi L. J. Rygl, ${ }^{86}$ Salvador Sánchez, ${ }^{107}$ David Sánchez-Arguelles,${ }^{66,67}$ Mahito Sasada, ${ }^{47,108}$ Tuomas Savolainen, ${ }^{109,110,7}$ F. Peter Schloerb, ${ }^{97}$ Karl-Friedrich Schuster, ${ }^{29}$ Lijing Shao, ${ }^{7,71}$ Zhiqiang Shen (沈志强), ${ }^{43,44}$ Des Small, ${ }^{79}$ Bong Won Sohn, ${ }^{33,34,111}$ Jason SooHoo, ${ }^{12}$ He Sun (孙赫), ${ }^{25}$ Fumie Tazaki, ${ }^{47}$ Alexandra J. Tetarenko, ${ }^{112}$ Paul Tiede,${ }^{31,32}$ Remo P. J. Tilanus, ${ }^{4,64,113,20}$ Michael Titus, ${ }^{12}$ Kenji Toma, ${ }^{114,115}$ Pablo Torne, ${ }^{7,107}$ Tyler Trent, ${ }^{20}{ }^{79}$ Efthalia Traianou, ${ }^{7}$ Sascha Trippe, ${ }^{116}$ Ilse van Bemmel ${ }_{119}^{79}$ Huib Jan van Langevelde, ${ }^{79,117}$ Daniel R. van Rossum, ${ }^{4}$ Jan Wagner, ${ }^{7}$ Derek Ward-Thompson, ${ }_{52}^{118}$ John Wardle, ${ }^{119}$ Jonathan Weintroub, ${ }^{5,6}$ Norbert Wex, ${ }^{7}$ Robert Wharton, ${ }^{7}$ Maciek Wielgus, ${ }^{5,6}$ George N. Wong, ${ }^{62}$ Qingwen Wu (吴庆文), ${ }^{120}$ Doosoo Yoon, ${ }^{42}$ André Young, ${ }^{4}$ Ken Young, ${ }_{43}$ Feng Yuan (袁峰), ${ }^{43,68,121}$ Ye-Fei Yuan (袁业飞), ${ }^{122}$ J. Anton Zensus, ${ }^{7}$ Guang-Yao Zhao, ${ }^{14}$ and Shan-Shan Zhao ${ }^{43}$

\section{(EHT Collaboration)}

\author{
${ }^{1}$ Institut für Theoretische Physik, Goethe-Universität, \\ Max-von-Laue-Strasse 1, 60438 Frankfurt, Germany \\ ${ }^{2}$ Frankfurt Institute for Advanced Studies, Ruth-Moufang-Strasse 1, 60438 Frankfurt, Germany \\ ${ }^{3}$ School of Mathematics, Trinity College, Dublin 2, Ireland \\ ${ }^{4}$ Department of Astrophysics, Institute for Mathematics, Astrophysics and Particle Physics (IMAPP), \\ Radboud University, P.O. Box 9010, 6500 GL Nijmegen, Netherlands \\ ${ }^{5}$ Black Hole Initiative at Harvard University, 20 Garden Street, Cambridge, Massachusetts 02138, USA
}




\author{
${ }^{6}$ Center for Astrophysics-Harvard \& Smithsonian, \\ 60 Garden Street, Cambridge, Massachusetts 02138, USA \\ ${ }^{7}$ Max-Planck-Institut für Radioastronomie, Auf dem Hügel 69, D-53121 Bonn, Germany \\ ${ }^{8}$ Tsung-Dao Lee Institute and School of Physics and Astronomy, Shanghai Jiao Tong University, \\ Shanghai, 200240, China \\ ${ }^{9}$ Institut für Theoretische Physik, Goethe-Universität Frankfurt, Max-von-Laue-Straße 1, \\ D-60438 Frankfurt am Main, Germany \\ ${ }^{10}$ Department of Physics, National and Kapodistrian University of Athens, \\ Panepistimiopolis, GR 15783 Zografos, Greece \\ ${ }^{11}$ Mullard Space Science Laboratory, University College London, Holmbury St. Mary, \\ Dorking, Surrey, RH5 6NT, United Kingdom \\ ${ }^{12}$ Massachusetts Institute of Technology Haystack Observatory, \\ 99 Millstone Road, Westford, Massachusetts 01886, USA \\ ${ }^{13}$ National Astronomical Observatory of Japan, 2-21-1 Osawa, Mitaka, Tokyo 181-8588, Japan \\ ${ }^{14}$ Instituto de Astrofísica de Andalucía-CSIC, Glorieta de la Astronomía s/n, E-18008 Granada, Spain \\ ${ }^{15}$ Department of Physics, Faculty of Science, University of Malaya, 50603 Kuala Lumpur, Malaysia \\ ${ }^{16}$ Center for Computational Astrophysics, Flatiron Institute, \\ 162 Fifth Avenue, New York, New York 10010, USA \\ ${ }^{17}$ Institute of Astronomy and Astrophysics, Academia Sinica, $11 \mathrm{~F}$ of Astronomy-Mathematics Building, \\ AS/NTU No. 1, Sec. 4, Roosevelt Rd, Taipei 10617, Taiwan, R.O.C. \\ ${ }^{18}$ Departament d'Astronomia i Astrofísica, Universitat de València, \\ C. Dr. Moliner 50, E-46100 Burjassot, València, Spain \\ ${ }^{19}$ Observatori Astronòmic, Universitat de València, \\ C. Catedrático José Beltrán 2, E-46980 Paterna, València, Spain \\ ${ }^{20}$ Steward Observatory and Department of Astronomy, University of Arizona, \\ 933 N. Cherry Avenue, Tucson, Arizona 85721, USA \\ ${ }^{21}$ Fermi National Accelerator Laboratory, MS209, P.O. Box 500, Batavia, Illinois 60510, USA \\ ${ }^{22}$ Department of Astronomy and Astrophysics, University of Chicago, \\ 5640 South Ellis Avenue, Chicago, Illinois 60637, USA \\ ${ }^{23}$ East Asian Observatory, 660 N. A'ohoku Place, Hilo, Hawaii 96720, USA \\ ${ }^{24}$ Nederlandse Onderzoekschool voor Astronomie (NOVA), PO Box 9513, 2300 RA Leiden, Netherlands \\ ${ }^{25}$ California Institute of Technology, 1200 East California Boulevard, Pasadena, California 91125, USA \\ ${ }^{26}$ Institute of Astronomy and Astrophysics, Academia Sinica, 645 N. A'ohoku Place, Hilo, Hawaii 96720, USA \\ ${ }^{27}$ Department of Physics, McGill University, 3600 rue University, \\ Montréal, Quebec City H3A 2T8, Canada \\ ${ }^{28}$ McGill Space Institute, McGill University, 3550 rue University, \\ Montréal, Quebec City H3A 2A7, Canada \\ ${ }^{29}$ Institut de Radioastronomie Millimétrique, 300 rue de la Piscine, F-38406 Saint Martin d'Hères, France \\ ${ }^{30}$ Perimeter Institute for Theoretical Physics, 31 \\ Caroline Street North, Waterloo, Ontario, N2L 2Y5, Canada \\ ${ }^{31}$ Department of Physics and Astronomy, University of Waterloo, \\ 200 University Avenue West, Waterloo, Ontario, N2L 3G1, Canada \\ ${ }^{32}$ Waterloo Centre for Astrophysics, University of Waterloo, Waterloo, Ontario, N2L 3G1, Canada \\ ${ }^{33}$ Korea Astronomy and Space Science Institute, Daedeok-daero 776, \\ Yuseong-gu, Daejeon 34055, Republic of Korea \\ ${ }^{34}$ University of Science and Technology, Gajeong-ro 217, Yuseong-gu, Daejeon 34113, Republic of Korea \\ ${ }^{35}$ Kavli Institute for Cosmological Physics, University of Chicago, \\ 5640 South Ellis Avenue, Chicago, Illinois 60637, USA \\ ${ }^{36}$ Department of Physics, University of Chicago, 5720 South Ellis Avenue, Chicago, Illinois 60637, USA \\ ${ }^{37}$ Enrico Fermi Institute, University of Chicago, 5640 South Ellis Avenue, Chicago, Illinois 60637, USA \\ ${ }^{38}$ Princeton Center for Theoretical Science, Jadwin Hall, Princeton University, \\ Princeton, New Jersey 08544, USA \\ ${ }^{39}$ NASA Hubble Fellowship Program, Einstein Fellow \\ ${ }^{40}$ Data Science Institute, University of Arizona, 1230 N. Cherry Avenue, Tucson, Arizona 85721, USA \\ ${ }^{41}$ Cornell Center for Astrophysics and Planetary Science, Cornell University, \\ Ithaca, New York 14853, USA \\ ${ }^{42}$ Anton Pannekoek Institute for Astronomy, University of Amsterdam, \\ Science Park 904, 1098 XH, Amsterdam, Netherlands \\ ${ }^{43}$ Shanghai Astronomical Observatory, Chinese Academy of Sciences, \\ 80 Nandan Road, Shanghai 200030, People's Republic of China
}


${ }^{44}$ Key Laboratory of Radio Astronomy, Chinese Academy of Sciences, Nanjing 210008, People's Republic of China

${ }^{45}$ Physics Department, Fairfield University, 1073 North Benson Road, Fairfield, Connecticut 06824, USA

${ }^{46}$ Department of Space, Earth and Environment, Chalmers University of Technology, Onsala Space Observatory, SE-43992 Onsala, Sweden

${ }^{47}$ Mizusawa VLBI Observatory, National Astronomical Observatory of Japan, 2-12 Hoshigaoka, Mizusawa, Oshu, Iwate 023-0861, Japan

${ }^{48}$ Department of Astronomical Science, The Graduate University for Advanced Studies (SOKENDAI), 2-21-1 Osawa, Mitaka, Tokyo 181-8588, Japan

${ }^{49}$ Department of Astronomy and Columbia Astrophysics Laboratory, Columbia University, 550 W 120th Street, New York, New York 10027, USA

${ }^{50}$ Dipartimento di Fisica "E. Pancini", Universitá di Napoli "Federico II", Compl. Univ. di Monte S. Angelo, Edificio G, Via Cinthia, I-80126, Napoli, Italy

${ }^{51}$ INFN Sez. di Napoli, Compl. Univ. di Monte S. Angelo, Edificio G, Via Cinthia, I-80126, Napoli, Italy

${ }^{52}$ Wits Centre for Astrophysics, University of the Witwatersrand, 1 Jan Smuts Avenue, Braamfontein, Johannesburg 2050, South Africa

${ }^{53}$ Department of Physics, University of Pretoria, Hatfield, Pretoria 0028, South Africa

${ }^{54}$ Centre for Radio Astronomy Techniques and Technologies,

Department of Physics and Electronics, Rhodes University, Makhanda 6140, South Africa

${ }^{55}$ LESIA, Observatoire de Paris, Université PSL, CNRS, Sorbonne Université,

Université de Paris, 5 place Jules Janssen, 92195 Meudon, France

${ }^{56}$ National Astronomical Observatories, Chinese Academy of Sciences, 20A Datun Road, Chaoyang District, Beijing 100101, PR China

${ }^{57}$ University of Massachusetts Boston,

100 William T. Morrissey Boulevard, Boston, Massachusetts 02125, USA

${ }^{58}$ National Radio Astronomy Observatory, 520 Edgemont Road, Charlottesville, Virginia 22903, USA

${ }^{59}$ Steward Observatory and Department of Astronomy, University of Arizona, 933 North Cherry Avenue, Tucson, Arizona 85721, USA

${ }^{60}$ Department of History of Science, Harvard University, Cambridge, Massachusetts 02138, USA

${ }^{61}$ Department of Physics, Harvard University, Cambridge, Massachusetts 02138, USA

${ }^{62}$ Department of Physics, University of Illinois, 1110 West Green Street, Urbana, Illinois 61801, USA

${ }^{63}$ Department of Astronomy, University of Illinois at Urbana-Champaign, 1002 West Green Street, Urbana, Illinois 61801, USA

${ }^{64}$ Leiden Observatory-Allegro, Leiden University, P.O. Box 9513, 2300 RA Leiden, Netherlands

${ }^{65}$ CP3-Origins, University of Southern Denmark, Campusvej 55, DK-5230 Odense M, Denmark

${ }^{66}$ Instituto Nacional de Astrofísica, Óptica y Electrónica. Apartado Postal 51 y 216, 72000. Puebla Pue., México

${ }^{67}$ Consejo Nacional de Ciencia y Tecnología, Av. Insurgentes Sur 1582, 03940, Ciudad de México, México

${ }^{68}$ Key Laboratory for Research in Galaxies and Cosmology, Chinese Academy of Sciences, Shanghai 200030, People's Republic of China

${ }^{69}$ NOVA Sub-mm Instrumentation Group, Kapteyn Astronomical Institute, University of Groningen, Landleven 12, 9747 AD Groningen, Netherlands

${ }^{70}$ Department of Astronomy, School of Physics, Peking University, Beijing 100871, People's Republic of China

${ }^{71}$ Kavli Institute for Astronomy and Astrophysics, Peking University, Beijing 100871, People's Republic of China

${ }^{72}$ Department of Astronomy, Graduate School of Science, The University of Tokyo, 7-3-1 Hongo, Bunkyo-ku, Tokyo 113-0033, Japan

${ }^{73}$ The Institute of Statistical Mathematics, 10-3 Midori-cho, Tachikawa, Tokyo, 190-8562, Japan

${ }^{74}$ Department of Statistical Science, The Graduate University for Advanced Studies (SOKENDAI), 10-3 Midori-cho, Tachikawa, Tokyo 190-8562, Japan

${ }^{75}$ Kavli Institute for the Physics and Mathematics of the Universe, The University of Tokyo, 5-1-5 Kashiwanoha, Kashiwa, 277-8583, Japan

${ }^{76}$ Institute for Astrophysical Research, Boston University, 725 Commonwealth New Jersey, Boston, Massachusetts 02215, USA

${ }^{77}$ Astronomical Institute, St. Petersburg University, Universitetskij pr., 28, Petrodvorets, 198504 St.Petersburg, Russia

${ }^{78}$ Institute for Cosmic Ray Research, The University of Tokyo, 5-1-5 Kashiwanoha, Kashiwa, Chiba 277-8582, Japan

${ }^{79}$ Joint Institute for VLBI ERIC (JIVE), Oude Hoogeveensedijk 4, 7991 PD Dwingeloo, Netherlands 
${ }^{80}$ Kogakuin University of Technology \& Engineering, Academic Support Center, 2665-1 Nakano, Hachioji, Tokyo 192-0015, Japan

${ }^{81}$ Physics Department, National Sun Yat-Sen University,

No. 70, Lien-Hai Rd, Kaosiung City 80424, Taiwan, R.O.C

${ }^{82}$ National Optical Astronomy Observatory, 950 N. Cherry Avenue, Tucson, Arizona 85719, USA

${ }^{83}$ Key Laboratory for Particle Astrophysics, Institute of High Energy Physics,

Chinese Academy of Sciences, 19B Yuquan Road, Shijingshan District, Beijing, People's Republic of China

${ }^{84}$ School of Astronomy and Space Science, Nanjing University,

Nanjing 210023, People's Republic of China

${ }^{85}$ Key Laboratory of Modern Astronomy and Astrophysics, Nanjing University,

Nanjing 210023, People's Republic of China

${ }^{86}$ Italian ALMA Regional Centre, INAF-Istituto di Radioastronomia, Via P. Gobetti 101, I-40129 Bologna, Italy

${ }^{87}$ Department of Physics, National Taiwan University,

No.1, Sect.4, Roosevelt Road., Taipei 10617, Taiwan, R.O.C

${ }^{88}$ Instituto de Radioastronomía y Astrofísica, Universidad Nacional Autónoma de México, Morelia 58089, México

${ }^{89}$ Instituto de Astronomía, Universidad Nacional Autónoma de México, CdMx 04510, México

${ }^{90}$ Yunnan Observatories, Chinese Academy of Sciences,

650011 Kunming, Yunnan Province, People's Republic of China

${ }^{91}$ Center for Astronomical Mega-Science, Chinese Academy of Sciences,

20A Datun Road, Chaoyang District, Beijing, 100012, People's Republic of China

${ }^{92}$ Key Laboratory for the Structure and Evolution of Celestial Objects, Chinese Academy of Sciences, 650011 Kunming, People's Republic of China

${ }^{93}$ Gravitation Astroparticle Physics Amsterdam (GRAPPA) Institute, University of Amsterdam,

Science Park 904, 1098 XH Amsterdam, Netherlands

${ }^{94}$ School of Natural Sciences, Institute for Advanced Study, 1 Einstein Drive, Princeton, New Jersey 08540, USA

${ }^{95}$ Astronomy Department, Universidad de Concepción, Casilla 160-C, Concepción, Chile

${ }^{96}$ National Institute of Technology, Hachinohe College,

16-1 Uwanotai, Tamonoki, Hachinohe City, Aomori 039-1192, Japan

${ }^{97}$ Department of Astronomy, University of Massachusetts, 01003, Amherst, Massachusetts, USA

${ }^{98}$ South African Radio Astronomy Observatory, Observatory 7925, Cape Town, South Africa

${ }^{99}$ Villanova University, Mendel Science Center Rm. 263B,

800 E Lancaster Avenue, Villanova Pennsylvania 19085

${ }^{100}$ Physics Department, Washington University CB 1105, St Louis, Missouri 63130, USA

${ }^{101}$ Canadian Institute for Theoretical Astrophysics, University of Toronto,

60 St. George Street, Toronto, Ontario M5S 3H8, Canada

${ }^{102}$ Dunlap Institute for Astronomy and Astrophysics, University of Toronto,

50 St. George Street, Toronto, Ontario M5S 3H4, Canada

${ }^{103}$ Canadian Institute for Advanced Research, 180 Dundas St West, Toronto, Ontario M5G 1Z8, Canada

${ }^{104}$ Radio Astronomy Laboratory, University of California, Berkeley, California 94720, USA

${ }^{105}$ Department of Physics, National Taiwan Normal University,

No. 88, Sec. 4, Tingzhou Road, Taipei 116, Taiwan, R.O.C.

${ }^{106}$ Department of Astrophysical Sciences, Peyton Hall, Princeton University, Princeton, New Jersey 08544, USA

${ }^{107}$ Instituto de Radioastronomía Milimétrica, IRAM,

Avenida Divina Pastora 7, Local 20, E-18012, Granada, Spain

${ }^{108}$ Hiroshima Astrophysical Science Center, Hiroshima University,

1-3-1 Kagamiyama, Higashi-Hiroshima, Hiroshima 739-8526, Japan

${ }^{109}$ Aalto University Department of Electronics and Nanoengineering, PL 15500, FI-00076 Aalto, Finland

${ }^{110}$ Aalto University Metsähovi Radio Observatory, Metsähovintie 114, FI-02540 Kylmälä, Finland

${ }^{111}$ Department of Astronomy, Yonsei University, Yonsei-ro 50, Seodaemun-gu, 03722 Seoul, Republic of Korea

${ }^{112}$ East Asian Observatory, 660 North A'ohoku Place, Hilo, Hawaii 96720, USA

${ }^{113}$ Netherlands Organisation for Scientific Research (NWO),

Postbus 93138, 2509 AC Den Haag, Netherlands

${ }^{114}$ Frontier Research Institute for Interdisciplinary Sciences, Tohoku University, Sendai 980-8578, Japan

${ }^{115}$ Astronomical Institute, Tohoku University, Sendai 980-8578, Japan 


\author{
${ }^{116}$ Department of Physics and Astronomy, Seoul National University, \\ Gwanak-gu, Seoul 08826, Republic of Korea \\ ${ }^{117}$ Leiden Observatory, Leiden University, Postbus 2300, 9513 RA Leiden, Netherlands \\ ${ }^{118}$ Jeremiah Horrocks Institute, University of Central Lancashire, Preston PR1 2HE, United Kingdom \\ ${ }^{119}$ Physics Department, Brandeis University, 415 South Street, Waltham, Massachusetts 02453, USA \\ ${ }^{120}$ School of Physics, Huazhong University of Science and Technology, \\ Wuhan, Hubei, 430074, People's Republic of China \\ ${ }^{121}$ School of Astronomy and Space Sciences, University of Chinese Academy of Sciences, \\ No. 19A Yuquan Road, Beijing 100049, People's Republic of China \\ ${ }^{122}$ Astronomy Department, University of Science and Technology of China, \\ Hefei 230026, People's Republic of China
}

(Received 29 November 2020; accepted 21 April 2021; published 20 May 2021)

\begin{abstract}
Our understanding of strong gravity near supermassive compact objects has recently improved thanks to the measurements made by the Event Horizon Telescope (EHT). We use here the M87* shadow size to infer constraints on the physical charges of a large variety of nonrotating or rotating black holes. For example, we show that the quality of the measurements is already sufficient to rule out that M87* is a highly charged dilaton black hole. Similarly, when considering black holes with two physical and independent charges, we are able to exclude considerable regions of the space of parameters for the doubly-charged dilaton and the Sen black holes.
\end{abstract}

DOI: $10.1103 /$ PhysRevD.103.104047

\section{INTRODUCTION}

General relativity (GR) was formulated to consistently account for the interaction of dynamical gravitational fields with matter and energy, the central idea of which is that the former manifests itself through modifications of spacetime geometry and is fully characterized by a metric tensor. While the physical axioms that GR is founded on are contained in the equivalence principle [1,2], the EinsteinHilbert action further postulates that the associated equations of motion involve no more than second-order derivatives of the metric tensor.

The strength of the gravitational field outside an object of mass $M$ and characteristic size $R$, in geometrized units $(G=c=1)$, is related to its compactness $\mathcal{C}:=M / R$, which is $\sim 10^{-6}$ for the Sun, and takes values $\sim 0.2-1$ for compact objects such as neutron stars and black holes. Predictions from GR have been tested and validated by various solar-system experiments to very high precision $[2,3]$, setting it on firm footing as the best-tested theory of classical gravity in the weak-field regime. It is important, however, to consider whether signatures of deviations from the Einstein-Hilbert action, e.g., due to higher derivative terms [4-6], could appear in measurements of phenomena occurring in strong-field regimes where $\mathcal{C}$ is large. Similarly, tests are needed to assess whether generic

Published by the American Physical Society under the terms of the Creative Commons Attribution 4.0 International license. Further distribution of this work must maintain attribution to the author(s) and the published article's title, journal citation, and DOI. violations of the equivalence principle occur in strongfields due, e.g., to the presence of additional dynamical fields, such as scalar [7,8] or vector fields [9-13], that may fall off asymptotically. Agreement with the predictions of GR coming from observations of binary pulsars [14-16], and of the gravitational redshift [17] and geodetic orbitprecession [18] of the star S2 near our galaxy's central supermassive compact object Sgr $\mathrm{A}^{\star}$ by the GRAVITY collaboration, all indicate the success of GR in describing strong-field physics as well. In addition, with the gravitational-wave detections of coalescing binaries of compact objects by the LIGO/Virgo collaboration $[19,20]$ and the first images of black holes produced by EHT, it is now possible to envision testing GR at the strongest field strengths possible.

While the inferred size of the shadow from the recently obtained horizon-scale images of the supermassive compact object in M87 galaxy by the EHT collaboration [2126] was found to be consistent to within $17 \%$ for a $68 \%$ confidence interval of the size predicted from GR for a Schwarzschild black hole using the a priori known estimates for the mass and distance of M87* based on stellar dynamics [27], this measurement admits other possibilities, as do various weak-field tests [2,28]. Since the number of alternative theories to be tested using this measurement is large, a systematic study of the constraints set by a strong-field measurement is naturally more tractable within a theory-agnostic framework, and various such systems have recently been explored [29-36]. This approach allows for tests of a broad range of possibilities that may not be captured in the limited set of known solutions. This was exploited in Ref. [28], where 
constraints on two deformed metrics were obtained when determining how different M87* could be from a Kerr black hole while remaining consistent with the EHT measurements.

However, because such parametric tests cannot be connected directly to an underlying property of the alternative theory, here we use instead the EHT measurements to set bounds on the physical parameters, i.e., angular momentum, electric charge, scalar charge, etc.- - and which we will generically refer to as "charges" (or hairs) - that various well-known black-hole solutions depend upon. Such analyses can be very instructive [37-51] since they can shed light on which underlying theories are promising candidates and which must be discarded or modified. At the same time, they may provide insight into the types of additional dynamical fields that may be necessary for a complete theoretical description of physical phenomena, and whether associated violations of the equivalence principle occur.

More specifically, since the bending of light in the presence of curvature-either in static or in stationary spacetimes-is assured in any metric theory of gravity, and the presence of large amounts of mass in very small volumes can allow for the existence of a region where null geodesics move on spherical orbits, an examination of the characteristics of such photon regions, when they exist, is a useful first step. The projected asymptotic collection of the photons trajectories that are captured by the black hole -namely, all of the photon trajectories falling within the value of the impact parameter at the unstable circular orbit in the case of nonrotating black holes-will appear as a dark area to a distant observer and thus represents the "shadow" of the capturing compact object. This shadowwhich can obviously be associated with black holes [5257], but also more exotic compact objects such as gravastars $[58,59]$ or naked singularities $[60,61]$-is determined entirely by the underlying spacetime metric. Therefore, the properties of the shadow-and at lowest order its sizerepresent valuable observables common to all metric theories of gravity, and can be used to test them for their agreement with EHT measurements.

While the EHT measurement contains far more information related to the flow of magnetized plasma near M87*, we will consider only the measurement of the size of the bright ring. Here we consider various spherically symmetric blackhole solutions, from GR that are either singular (see, e.g., [62]) or non-singular [63-65], and string theory [66-70]. Additionally, we also consider the Reissner-Nordström (RN) and the Janis-Newman-Winicour (JNW) [71] naked singularity solutions, the latter being a solution of the EinsteinKlein-Gordon system. Many of these solutions have been recently summarized in Ref. [36], where they were cast in a generalized expansion of static and spherically symmetric metrics. Since angular momentum plays a key role in astrophysical scenarios, we also consider various rotating black-hole solutions [72-75] which can be expressed in the Newman-Janis form [76] to facilitate straightforward analytical computations. It is to be noted that this study is meant to be a proof of principle and that while the constraints we can set here are limited, the analytical procedure outlined below for this large class of metrics is general, so that as future observations become available, we expect the constraints that can be imposed following the approach proposed here to be much stronger.

\section{SPHERICAL NULL GEODESICS AND SHADOWS}

For all the static, spherically symmetric spacetimes we consider here, the definition of the shadow can be cast in rather general terms. In particular, for all the solutions considered, the line element expressed in areal-radial polar coordinates $(t, \tilde{r}, \theta, \phi)$ has the form ${ }^{1}$

$d s^{2}=g_{\mu \nu} d x^{\mu} d x^{\nu}=-f(\tilde{r}) \mathrm{d} t^{2}+\frac{g(\tilde{r})}{f(\tilde{r})} \mathrm{d} \tilde{r}^{2}+\tilde{r}^{2} \mathrm{~d} \Omega_{2}^{2}$,

and the photon region, which degenerates into a photon sphere, is located at $\tilde{r}=: \tilde{r}_{\mathrm{ps}}$, which can be obtained by solving [28]

$$
\tilde{r}-\frac{2 f(\tilde{r})}{\partial_{\tilde{r}} f(\tilde{r})}=0 .
$$

The boundary of this photon sphere when observed from the frame of an asymptotic observer, due to gravitational lensing, appears to be a circle of size [28]

$$
\tilde{r}_{\mathrm{sh}}=\frac{\tilde{r}_{\mathrm{ps}}}{\sqrt{f\left(\tilde{r}_{\mathrm{ps}}\right)}}
$$

On the other hand, the class of Newman-Janis stationary, axisymmetric spacetimes we consider here [76], which are geodesically integrable (see, e.g., $[55,77,78]$ ), can be expressed in Boyer-Lindquist coordinates $(t, r, \theta, \phi)$ as

$$
\begin{aligned}
\mathrm{d} s^{2}= & -f \mathrm{~d} t^{2}-2 a \sin ^{2} \theta(1-f) \mathrm{d} t \mathrm{~d} \phi \\
& +\left[\Sigma+a^{2} \sin ^{2} \theta(2-f)\right] \sin ^{2} \theta \mathrm{d} \phi^{2}+\frac{\Sigma}{\Delta} \mathrm{d} r^{2}+\Sigma \mathrm{d} \theta^{2},
\end{aligned}
$$

where $f=f(r, \theta)$ and $\Sigma(r, \theta):=r^{2}+a^{2} \cos ^{2} \theta$ and $\Delta(r):=\Sigma(r, \theta) f(r, \theta)+a^{2} \sin ^{2} \theta$. In particular, these are

\footnotetext{
${ }^{1}$ We use the tilde on the radial coordinate of static spacetimes to distinguish it from the corresponding radial coordinate of axisymmetric spacetimes.
} 
the stationary generalizations obtained by employing the Newman-Janis algorithm [76]) for "seed" metrics of the form (1) with $g(\tilde{r})=1$. $^{2}$

The Lagrangian $\mathcal{L}$ for geodesic motion in the spacetime (4) is given as $2 \mathcal{L}:=g_{\mu \nu} \dot{x}^{\mu} \dot{x}^{\nu}$, where an overdot represents a derivative with respect to the affine parameter, and $2 \mathcal{L}=$ -1 for timelike geodesics and $2 \mathcal{L}=0$ for null geodesics. The two Killing vectors $\partial_{t}$ and $\partial_{\phi}$ yield two constants of motion

$$
\begin{aligned}
-E & =-f \dot{t}-a \sin ^{2} \theta(1-f) \dot{\phi}, \\
L & =-a \sin ^{2} \theta(1-f) \dot{t}+\left[\Sigma+a^{2} \sin ^{2} \theta(2-f)\right] \sin ^{2} \theta \dot{\phi},
\end{aligned}
$$

in terms of which the geodesic equation for photons can be separated into

$$
\begin{aligned}
& \Sigma^{2} \dot{r}^{2}=\left(r^{2}+a^{2}-a \xi\right)^{2}-\Delta \mathcal{I}=: \mathcal{R}(r), \\
& \Sigma^{2} \dot{\theta}^{2}=\mathcal{I}-(a \sin \theta-\xi \csc \theta)^{2}=: \Theta(\theta),
\end{aligned}
$$

where we have introduced first $\xi:=L / E$, and then $\mathcal{I}:=\eta+(a-\xi)^{2}$. Also, $\eta$ is the Carter constant, and the existence of this fourth constant of motion is typically associated with the existence of an additional Killing-Yano tensor (see for example [56,80]).

In particular, we are interested here in spherical null geodesics (SNGs), which satisfy $\dot{r}=0$ and $\ddot{r}=0$ and are not necessarily planar; equivalently, SNGs can exist at locations where $\mathcal{R}(r)=0$ and $\mathrm{d} \mathcal{R}(r) / \mathrm{d} r=0$. Since these are only two equations in three variables $(r, \xi, \eta)$, it is convenient, for reasons that will become evident below, to obtain the associated conserved quantities along such SNGs in terms of their radii $r$ as (see also [81]),

$$
\begin{aligned}
\xi_{\mathrm{SNG}}(r) & =\frac{r^{2}+a^{2}}{a}-\frac{4 r \Delta}{a \partial_{r} \Delta}, \\
\eta_{\mathrm{SNG}}(r) & =\frac{r^{2}}{a^{2}\left(\partial_{r} \Delta\right)^{2}}\left[16 a^{2} \Delta-\left(r \partial_{r} \Delta-4 \Delta\right)^{2}\right] .
\end{aligned}
$$

The condition that $\Theta(\theta) \geq 0$, which must necessarily hold as can be seen from Eq. (7), restricts the radial range for which SNGs exist, and it is evident that this range depends on $\theta$. This region, which is filled by such SNGs, is called the photon region (see, e.g., Fig. 3.3 of [52]).

The equality $\Theta(\theta)=0$ determines the boundaries of the photon region, and the (disconnected) piece which lies in the exterior of the outermost horizon is of primary interest

\footnotetext{
${ }^{2}$ Note that while the Sen solution can be obtained via the Newman-Janis algorithm [79], the starting point is the static EMd-1 metric written in a non-areal-radial coordinate $\rho$ such that $g_{t t} g_{\rho \rho}=-1$.
}

since its image, as seen by an asymptotic observer, is the shadow. We denote the inner and outer surfaces of this photon region by $r_{\mathrm{p}-}(\theta)$ and $r_{\mathrm{p}+}(\theta)$ respectively, with the former (smaller) SNG corresponding to the location of a prograde photon orbit (i.e., $\xi_{\mathrm{SNG}}\left(r_{\mathrm{p}-}\right)>0$ ), and the latter to a retrograde orbit.

It can be shown that all of the SNGs that are admitted in the photon region, for both the spherically symmetric and axisymmetric solutions considered here, are unstable to radial perturbations. In particular, for the stationary solutions, the stability of SNGs at a radius $r=r_{\mathrm{SNG}}$ with respect to radial perturbations is determined by the sign of $\partial_{r}^{2} \mathcal{R}$, and when $\partial_{r}^{2} \mathcal{R}\left(r_{\mathrm{SNG}}\right)>$ 0 , SNGs at that radius are unstable. The expression for $\partial_{r}^{2} \mathcal{R}$ reads

$$
\partial_{r}^{2} \mathcal{R}=\frac{8 r}{\left(\partial_{r} \Delta\right)^{2}}\left[r\left(\partial_{r} \Delta\right)^{2}-2 r \Delta \partial_{r}^{2} \Delta+2 \Delta \partial_{r} \Delta\right]
$$

To determine the appearance of the photon region and the associated shadow, as seen by asymptotic observers, we can introduce the usual notion of celestial coordinates $(\alpha, \beta)$, which for any photon with constants of the motion $(\xi, \eta)$ can be obtained, for an asymptotic observer present at an inclination angle $i$ with respect to the spin-axis of the compact object as in [82]. For photons on an SNG, we can set the conserved quantities $(\xi, \eta)$ to the values given in Eq. (8) above to obtain [80,81]

$$
\begin{gathered}
\alpha_{\mathrm{sh}}=-\xi_{\mathrm{SNG}} \csc i \\
\beta_{\mathrm{sh}}= \pm\left(\eta_{\mathrm{SNG}}+a^{2} \cos ^{2} i-\xi_{\mathrm{SNG}}^{2} \cot ^{2} i\right)^{1 / 2} .
\end{gathered}
$$

Recognizing that $\beta= \pm \sqrt{\Theta(i)}$, it becomes clear that only the SNGs with $\Theta(i) \geq 0$ determine the apparent shadow shape. Since the photon region is not spherically symmetric in rotating spacetimes, the associated shadow is also not circular in general. It can be shown that the band of radii for which SNGs can exist narrows as we move away from the equatorial plane, and reduces to a single value at the pole, i.e., in the limit $\theta \rightarrow \pi / 2$, we have $r_{\mathrm{p}+}=r_{\mathrm{p}-}$ (see e.g., Fig. 3.3 of [52]). As a result, the parametric curve of the shadow boundary as seen by an asymptotic observer lying along the pole is perfectly circular, $\alpha_{\mathrm{sh}}^{2}+\beta_{\mathrm{sh}}^{2}=\eta_{\mathrm{SNG}}\left(r_{\mathrm{p} \pm, \pi / 2}\right)+$ $\xi_{\mathrm{SNG}}^{2}\left(r_{\mathrm{p} \pm, \pi / 2}\right)$.

We can now define the characteristic areal-radius of the shadow curve as [83]

$$
r_{\mathrm{sh}, A}:=\left(\frac{2}{\pi} \int_{r_{\mathrm{p}-}}^{r_{\mathrm{p}+}} \mathrm{d} r \beta_{\mathrm{sh}}(r) \partial_{r} \alpha_{\mathrm{sh}}(r)\right)^{1 / 2} .
$$




\section{SHADOW SIZE CONSTRAINTS FROM THE 2017 EHT OBSERVATIONS OF M87*}

Measurements of stellar dynamics near M87* were previously used to produce a posterior distribution function of the angular gravitational radius $\theta_{\mathrm{g}}:=M / D$, where $M$ is the mass of and $D$ the distance to M87*. The 2017 EHT observations of M87* can be similarly used to determine such a posterior [26]. These observations were used to determine the angular diameter $\hat{d}$ of the bright emission ring that surrounds the shadow [26]. In Sec. 5.3 there, using synthetic images from general-relativistic magnetohydrodynamics (GRMHD) simulations of accreting Kerr black holes for a wide range of physical scenarios, the scaling factor $\alpha=\hat{d} / \theta_{\mathrm{g}}$ was calibrated. For emission from the outermost boundary of the photon region of a Kerr black hole, $\alpha$ should lie in the range $\simeq 9.6-10.4$.

The EHT measurement picks out a class of best-fit images ("top-set") from the image library, with a mean value for $\alpha$ of 11.55 (for the "xs-ring" model) and 11.50 (for the "xs-ringauss" model), when using two different geometric crescent models for the images, implying that the geometric models were accounting for emission in the topset GRMHD images that preferentially fell outside of the photon ring. Using the distribution of $\alpha$ for these top-set images then enabled the determination of the posterior in the angular gravitational radius $P_{\text {obs }}\left(\theta_{\mathrm{g}}\right)$ for the EHT data. It is to be noted that this posterior was also determined using direct GRMHD fitting, and image domain feature extraction procedures, as described in Sec. 9.2 there, and a high level of consistency was found across all measurement methods. Finally, in Sec. 9.5 of [26], the fractional deviation in the angular gravitational radius $\delta$ was introduced as

$$
\delta:=\frac{\theta_{\mathrm{g}}}{\theta_{\mathrm{dyn}}}-1,
$$

where $\theta_{\mathrm{g}}$ and $\theta_{\mathrm{dyn}}$ were used to denote the EHT and the stellar-dynamics inferences of the angular gravitational radius, respectively. The posterior on $\delta$-as defined in Eq. (32) of [26] —was then obtained (see Fig. 21 there), and its width was found to be $\delta=-0.01 \pm 0.17$, for a $68 \%$ credible interval. This agreement of the 2017 EHT measurement of the angular gravitational radius for M87* with a previously existing estimate for the same, at much larger distances, constitutes a validation of the null hypothesis of the EHT, and in particular that M87* can be described by the Kerr black-hole solution.

Since the stellar dynamics measurements [27] are sensitive only to the monopole of the metric (i.e., the mass) due to negligible spin-dependent effects at the distances involved in that analysis, modeling M87* conservatively using the Schwarzschild solution is reasonable with their obtained posterior. Then, using the angular gravitational radius estimate from stellar dynamics yields a prediction for the angular shadow radius $\theta_{\mathrm{sh}}=r_{\mathrm{sh}} / D$ as being $\theta_{\mathrm{sh}}=$ $3 \sqrt{3} \theta_{\text {dyn }}$. The 2017 EHT measurement, which includes spin-dependent effects as described above and which probes near-horizon scales, then determines the allowed spread in the angular shadow diameter as, $\theta_{\mathrm{sh}} \approx$ $3 \sqrt{3}(1 \pm 0.17) \theta_{\mathrm{g}}$, at $68 \%$ confidence levels [28]. Finally, since both angular estimates $\theta_{\mathrm{sh}}$ and $\theta_{\mathrm{g}}$ make use of the same distance estimate to $\mathrm{M} 87^{*}$, it is possible to convert the $1-\sigma$ bounds on $\theta_{\mathrm{sh}}$ to bounds on the allowed shadow size for M87*.

That is, independently of whether the underlying solution be spherically symmetric (in which case we will consider $\left.\tilde{r}_{\mathrm{sh}}\right)$ or axisymmetric $\left(r_{\mathrm{sh}, A}\right)$, the shadow size of M87* must lie in the range $3 \sqrt{3}(1 \pm 0.17) M$ [28], i.e., (see gray-shaded region in Fig. 2)

$4.31 M \approx r_{\text {sh,EHT- } \min } \leq \tilde{r}_{\mathrm{sh}}, r_{\mathrm{sh}, A} \leq r_{\text {sh,EHT- } \max } \approx 6.08 M$,

where we have introduced the maximum/minimum shadow radii $r_{\text {sh,EHT-max }} / r_{\text {sh,EHT-min }}$ inferred by the EHT, at $68 \%$ confidence levels.

Note that the bounds thus derived are consistent with compact objects that cast shadows that are both significantly smaller and larger than the minimum and maximum shadow sizes that a Kerr black hole could cast, which lie in the range, $4.83 M-5.20 M$ (see, e.g., $[28,84]$ ).

An important caveat here is that the EHT posterior distribution on $\theta_{\mathrm{g}}$ was obtained after a comparison with a large library of synthetic images built from GRMHD simulations of accreting Kerr black holes [25]. Ideally, a rigorous comparison with non-Kerr solutions would require a similar analysis and posterior distributions built from equivalent libraries obtained from GRMHD simulations of such non-Kerr solutions. Besides being computationally unfeasible, this approach is arguably not necessary in practice. For example, the recent comparative analysis of Ref. [50] has shown that the image libraries produced in this way would be very similar and essentially indistinguishable, given the present quality of the observations. As a result, we adopt here the working assumption that the 1- $\sigma$ uncertainty in the shadow angular size for non-Kerr solutions is very similar to that for Kerr black holes, and hence employ the constraints (14) for all of the solutions considered here.

\section{NOTABLE PROPERTIES OF VARIOUS SPACETIMES}

As mentioned above, a rigorous comparison with nonKerr black holes would require constructing a series of exhaustive libraries of synthetic images obtained from GRMHD simulations on such non-Kerr black holes. In turn, this would provide consistent posterior distributions 
TABLE I. Summary of properties of spacetimes used here. For easy access, we show whether the spacetime contains a rotating compact object or not, whether it contains a spacetime singularity, and what type of stationary nongravitational fields are present in the spacetime. Starred spacetimes contain naked singularities and daggers indicate a violation of the equivalence principle (see, e.g., [36]); In particular, these indicate violations of the weak equivalence principle due to a varying fine structure constant, a result of the coupling of the dilaton to the EM Lagrangian [36,89].

\begin{tabular}{lccl}
\hline \hline Spacetime & Rotation & Singularity & \multicolumn{1}{c}{ Spacetime content } \\
\hline KN [73] & Yes & Yes & EM fields \\
Kerr [72] & Yes & Yes & vacuum \\
RN [62] & No & Yes & EM fields \\
RN $^{*}[62]$ & No & Yes & EM fields \\
Schwarzschild [62] & No & Yes & vacuum \\
Rot. Bardeen [75] & Yes & No & matter \\
Bardeen [63] & No & No & matter \\
Rot. Hayward [75] & Yes & No & matter \\
Frolov [65] & No & No & EM fields, matter \\
Hayward [64] & No & No & matter \\
JNW $^{*}[71]$ & No & Yes & scalar field \\
KS [66] & No & Yes & vacuum \\
Sen ${ }^{\dagger}[74]$ & Yes & Yes & EM, dilaton, axion fields \\
EMd- ${ }^{\dagger}[67,68]$ & No & Yes & EM, dilaton fields \\
EMd-2 ${ }^{\dagger}[70]$ & No & Yes & EM, EM, dilaton fields \\
\hline \hline
\end{tabular}

of angular gravitational radii for the various black holes and hence determine how $\delta$ varies across different non-Kerr black holes, e.g., for Sen black holes. Because this is computationally unfeasible - the construction of only the Kerr library has required the joint effort of several groups with the EHTC over a good fraction of a year-we briefly discuss below qualitative arguments to support our use of the bounds given in Eq. (14) above as an approximate, yet indicative, measure.

To this end, we summarize in Table I the relevant properties of the various solutions used here. First, we have considered here solutions from three types of theories, i.e., the underlying actions are either (a) Einstein-Hilbert-Maxwell-matter [62-66,71,72,75], (b) Einstein-Hilbert-Maxwell-dilaton-axion $[67,68,74]$, or (c) Einstein-Hilbert-Maxwell-Maxwell-dilaton [70]. This careful choice implies that the gravitational piece of the action is always given by the Einstein-Hilbert term and that matter is minimally coupled to gravity. As a result, the dynamical evolution of the accreting plasma is expected to be very similar to that in GR, as indeed found in Ref. [50]. Second, since a microphysical description that allows one to describe the interaction of the exotic matter present in some of the regular black-hole spacetimes used here $[63,64]$ - which typically do not satisfy some form of the energy conditions $[75,85]$ — with the ordinary matter is thus far lacking, it is reasonable to assume that the interaction between these two types of fluids is gravitational only. This is indeed what is done in standard numerical simulations, either in dynamical spacetimes (see, e.g., [86]), or in fixed ones [49,87]. Third, since the mass-energy in the matter and electromagnetic fields for the non-vacuum spacetimes used here is of the order of the mass of the central compact object $M$, while the total mass of the accreting plasma in the GRMHD simulations is only a tiny fraction of the same, it is reasonable to treat the spacetime geometry and the stationary fields as unaffected by the plasma. Fourth, we have also been careful not to use solutions from theories with modified electrodynamics (such as nonlinear electrodynamics). As a result, the electromagnetic Lagrangian in all of the theories considered here is the Maxwell Lagrangian (see, e.g., the discussion in [36] and compare with [53]). This ensures that in these spacetimes light moves along the null geodesics of the metric tensor (see, e.g., Sec. 4.3 of [62] and compare against Sec. 2 of [88]). Therefore, we are also assured that ray-tracing the radiation emitted from the accreting matter in these spacetimes can be handled similarly as in the Kerr spacetime.

Finally, under the assumption that the dominant effects in determining the angular gravitational radii come from variations in the location of the photon region and in location of the inner edge of the accretion disk in these spacetimes, it is instructive to learn how these two physical quantities vary when changing physical charges, and, in particular, to demonstrate that they are quantitatively comparable to the corresponding values for the Kerr spacetime.

For this purpose, we study the single-charge solutions used here and report in Fig. 1 the variation in the location of the photon spheres (left panel) and innermost stable circular orbit (ISCO) radii (right panel) as a function of the relevant physical charge (cf. left panel of Fig. 1 in the main text). Note that both the photon-sphere and the ISCO radii 

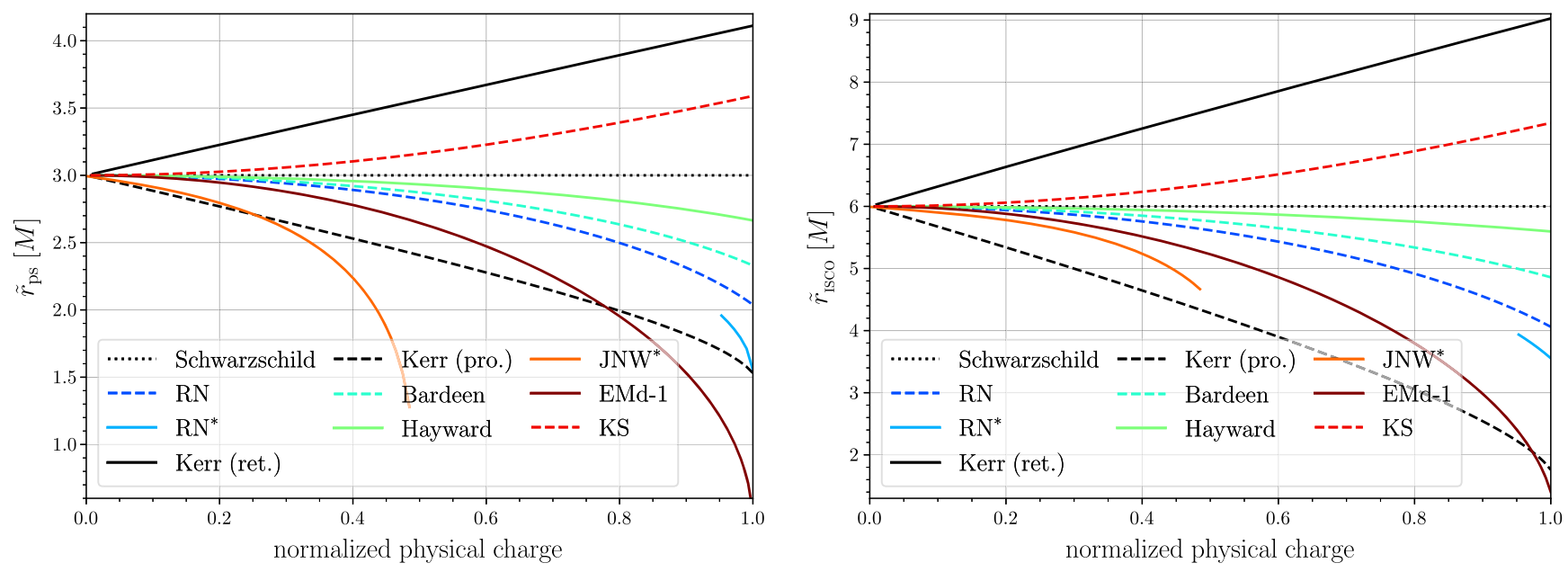

FIG. 1. Left: variation in the photon sphere radii for the single-charge nonrotating solutions as a function of the normalized physical charge. Right: The same as in the left panel but for the ISCO radii. We include also, for comparison, the variation in the Kerr equatorial prograde and retrograde photon sphere and ISCO radii in the left and right panels respectively.

depend exclusively on the $g_{t t}$ component of the metric when expressed using an areal radial coordinate $\tilde{r}$ (see, e.g., $[28,36])$. To gauge the effect of spin, we also show the variation in the locations of the equatorial prograde and retrograde circular photon orbits and the ISCOs in the Kerr black-hole spacetime, expressed in terms of the Cartesian Kerr-Schild radial coordinate $r_{\mathrm{CKS}}$, which, in the equatorial plane, is related to the Boyer-Lindquist radial coordinate used elsewhere in this work $r$ simply via [90]

$$
r_{\mathrm{CKS}}=\sqrt{r^{2}+a^{2}}
$$

It is apparent from Fig. 1 that the maximum deviation in the photon-sphere size from the Schwarzschild solution occurs for the EMd-1 black hole and is $\approx 75 \%$, while the size of the prograde equatorial circular photon orbit for Kerr deviates by at most $\approx 50 \%$. Similarly, the maximum variation in the ISCO size also occurs for the EMd-1 solution and is $\approx 73 \%$, while the prograde equatorial ISCO for Kerr can differ by $\approx 66 \%$.

\section{CHARGE CONSTRAINTS FROM THE EHT M87* OBSERVATIONS}

We first consider compact objects with a single "charge," and report in the left panel of Fig. 2 the variation in the shadow radius for various spherically symmetric black hole solutions, as well as for the RN and JNW naked singularities. $^{3}$ More specifically, we consider the black-hole

\footnotetext{
${ }^{3}$ While the electromagnetic and scalar charge parameters for the RN and JNW spacetimes are allowed to take values $\bar{q}>1$ and $0<\hat{\bar{\nu}}:=1-\bar{\nu}<1$ respectively, they do not cast shadows for $\bar{q}>$ $\sqrt{9 / 8}$ and $0.5 \leq \hat{\bar{\nu}}<1$ (see, e.g., Sec. IV D of [36] and references therein).
}

solutions given by Reissner-Nordström (RN) [62], Bardeen [63,75], Hayward [64,91], Kazakov-Solodhukin (KS) [66], and also the asymptotically-flat EinsteinMaxwell-dilaton (EMd-1) with $\phi_{\infty}=0$ and $\alpha_{1}=1$ $[67,68,88]$ solution (see Sec. IV of [36] for further details on these solutions). For each of these solutions we vary the corresponding charge (in units of $M$ ) in the allowed range, i.e., RN: $0<\bar{q} \leq 1$; Bardeen: $0<\bar{q}_{m} \leq \sqrt{16 / 27}$; Hayward: $0<\bar{l} \leq \sqrt{16 / 27}$; Frolov: $0<\bar{l} \leq \sqrt{16 / 27}$, $0<\bar{q} \leq 1$; KS: $0<\bar{l}$; EMd-1: $0<\bar{q}<\sqrt{2}$, but report the normalized value in the figure so that all curves are in a range between 0 and 1 . The figure shows the variation in the shadow size of KS black holes over the parameter range $0<\bar{l}<\sqrt{2}$. Note that the shadow radii tend to become smaller with increasing physical charge, but also that this is not universal behavior, since the KS black holes have increasing shadow radii (the singularity is smeared out on a surface for this solution, which increases in size with increasing $\bar{l}$ ).

Overall, it is apparent that the regular Bardeen, Hayward, and Frolov black-hole solutions are compatible with the present constraints. At the same time, the ReissnerNordström and Einstein-Maxwell-dilaton 1 black-hole solutions, for certain values of the physical charge, produce shadow radii that lie outside the 1- $\sigma$ region allowed by the 2017 EHT observations, and we find that these solutions are now constrained to take values in, $0<\bar{q} \lesssim 0.90$ and $0<$ $\bar{q} \lesssim 0.95$ respectively. Furthermore, the Reissner-Nordström naked singularity is entirely eliminated as a viable model for M87* and the Janis-Newman-Winicour naked singularity parameter space is restricted further by this measurement to $0<\hat{\bar{\nu}} \lesssim 0.47$. Finally, we also find that the KS black hole is also restricted to have charges in the range $\bar{l}<1.53$. In addition, note that the nonrotating Einstein-Maxwelldilaton 2 (EMd-2) solution [70]—which depends on two 

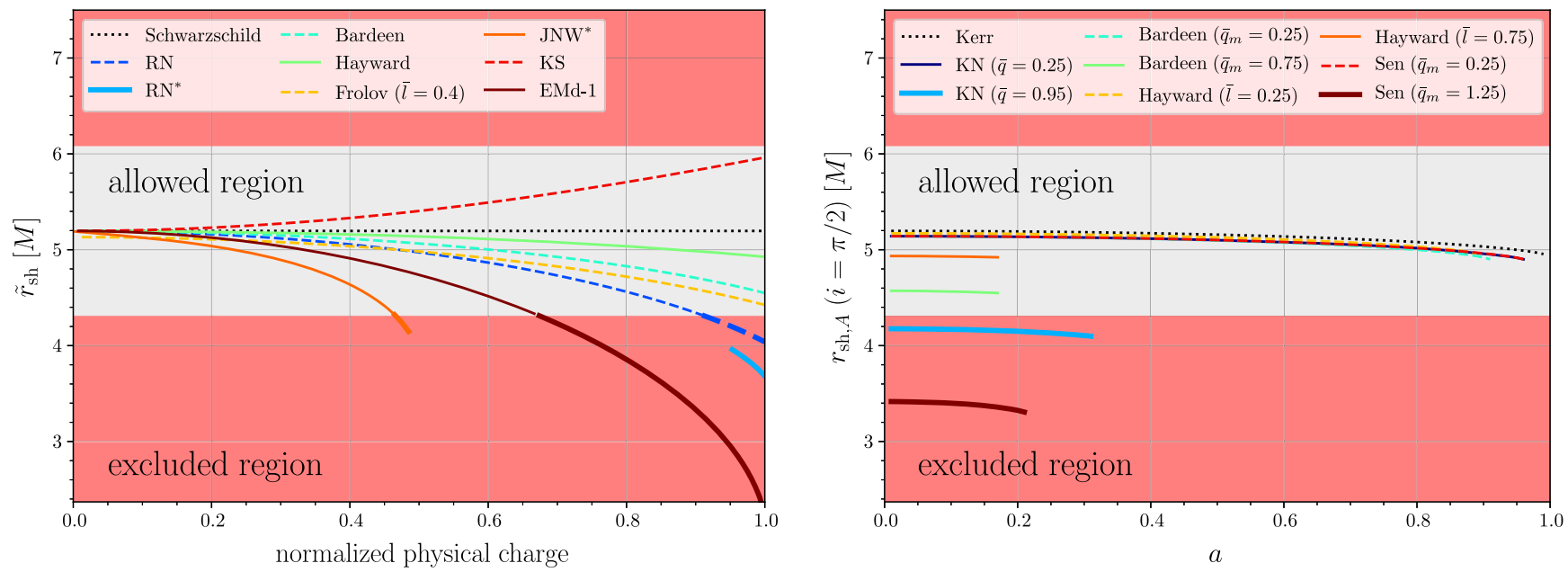

FIG. 2. Left: shadow radii $\tilde{r}_{\text {sh }}$ for various spherically symmetric black-hole solutions, as well as for the JNW and RN naked singularities (marked with an asterisk), as a function of the physical charge normalized to its maximum value. The gray/red shaded regions refer to the areas that are 1- $\sigma$ consistent/inconsistent with the 2017 EHT observations and highlight that the latter set constraints on the physical charges (see also Fig. 3 for the EMd-2 black hole). Right: shadow areal radii $r_{\mathrm{sh}, A}$ as a function of the dimensionless spin $a$ for four families of black-hole solutions when viewed on the equatorial plane $(i=\pi / 2)$. Also in this case, the observations restrict the ranges of the physical charges of the Kerr-Newman and the Sen black holes (see also Fig. 3).

independent charges — can also produce shadow radii that are incompatible with the EHT observations; we will discuss this further below. The two EMd black-hole solutions (1 and 2) correspond to fundamentally different field contents, as discussed in [70].

We report in the right panel of Fig. 2 the shadow areal radius $r_{\mathrm{sh}, A}$ for a number of stationary black holes, such as Kerr [72], Kerr-Newman (KN) [73], Sen [74], and the rotating versions of the Bardeen and Hayward black holes [75]. The data refers to an observer inclination angle of $i=\pi / 2$, and we find that the variation in the shadow size with spin at higher inclinations (of up to $i=\pi / 100$ ) is at most about $7.1 \%$ (for $i=\pi / 2$, this is $5 \%$ ); of course, at zero-spin the shadow size does not change with inclination. The shadow areal radii are shown as a function of the dimensionless spin of the black hole $a:=J / M^{2}$, where $J$ is its angular momentum, and for representative values of the additional parameters that characterize the solutions. Note that-similar to the angular momentum for a Kerr black hole-the role of an electric charge or the presence of a de Sitter core (as in the case of the Hayward black holes) is to reduce the apparent size of the shadow. Furthermore, on increasing the spin parameter, we recover the typical trend that the shadow becomes increasingly noncircular, as encoded, e.g., in the distortion parameter $\delta_{\text {sh }}$ defined in [57,83] (see Appendix). Also in this case, while the regular rotating Bardeen and Hayward solutions are compatible with the present constraints set by the 2017 EHT observations, the Kerr-Newman and Sen families of black holes can produce shadow areal radii that lie outside of the 1- $\sigma$ region allowed by the observations.
To further explore the constraints on the excluded regions for the Einstein-Maxwell-dilaton 2 and the Sen black holes, we report in Fig. 3 the relevant ranges for these two solutions. The Einstein-Maxwell-dilaton 2 black holes are nonrotating but have two physical charges expressed by the coefficients $0<\bar{q}_{e}<\sqrt{2}$ and $0<\bar{q}_{m}<\sqrt{2}$, while the Sen black holes spin $(a)$ and have an additional electromagnetic charge $\bar{q}_{m}$. Also in this case, the gray/red shaded regions refer to the areas that are consistent/inconsistent with the 2017 EHT observations. The figure shows rather easily that for these two black-hole families there are large

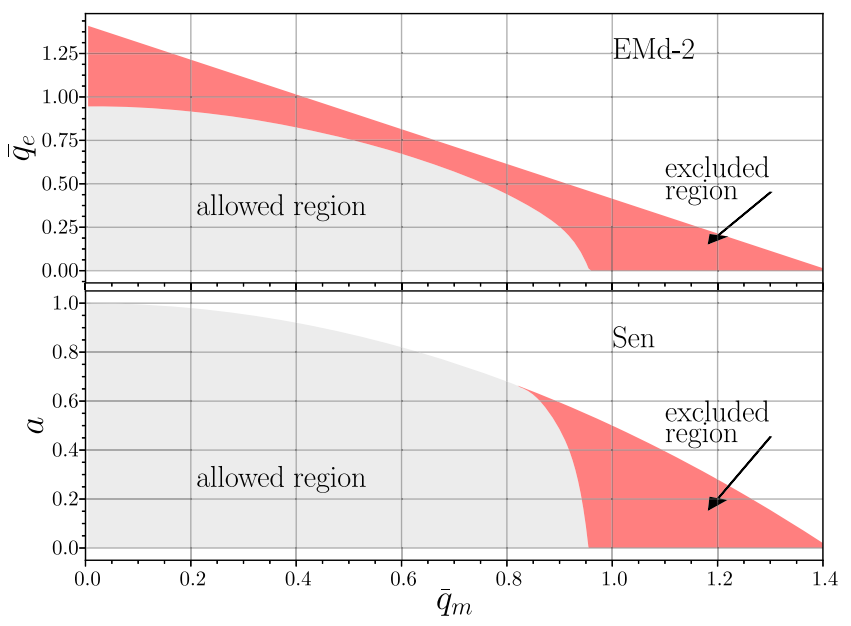

FIG. 3. Constraints set by the 2017 EHT observations on the nonrotating Einstein-Maxwell-dilaton 2 and on the rotating Sen black holes. Also in this case, the gray/red shaded regions refer to the areas that are 1- $\sigma$ consistent/inconsistent with the 2017 EHT observations). 
areas of the space of parameters that are excluded at the 1- $\sigma$ level. Not surprisingly, these areas are those where the physical charges take their largest values and hence the corresponding black-hole solutions are furthest away from the corresponding Schwarzschild or Kerr solutions. The obvious prospect is of course that as the EHT increases the precision of its measurements, increasingly larger portions of the space of parameters of these black holes will be excluded. Furthermore, other solutions that are presently still compatible with the observations may see their corresponding physical charges restricted.

\section{CONCLUSIONS}

As our understanding of gravity under extreme regimes improves, and as physical measurements of these regimes are now becoming available - either through the imaging of supermassive black holes or the detection of gravitational waves from stellar-mass black holes-we are finally in the position of setting some constraints to the large landscape of non-Kerr black holes that have been proposed over the years. We have used here the recent 2017 EHT observations of M87* to set constraints, at the 1- $\sigma$-level, on the physical charges - either electric, scalar, or angular momentum —of a large variety of static (nonrotating) or stationary (rotating) black holes.

In this way, when considering nonrotating black holes with a single physical charge, we have been able to rule out, at $68 \%$ confidence levels, the possibility that M87* is a near-extremal Reissner-Nordström or Einstein-Maxwelldilaton 1 black hole and that the corresponding physical charge must be in the range, RN: $0<\bar{q} \lesssim 0.90$ and EMd-1: $0<\bar{q} \lesssim 0.95$. We also find that it cannot be a ReissnerNordström naked singularity or a JNW naked singularity with large scalar charge, i.e., only $0<\hat{\bar{\nu}} \lesssim 0.47$ is allowed. Similarly, when considering black holes with two physical charges (either nonrotating or rotating), we have been able to exclude, with $68 \%$ confidence, considerable regions of the space of parameters in the case of the EinsteinMaxwell-dilaton 2, Kerr-Newman and Sen black holes. Although the idea of setting such constraints is an old one (see, e.g., [29-36,51,54,55]), and while there have been recent important developments in the study of other possible observational signatures of such alternative solutions, such as in X-ray spectra of accreting black holes (see, e.g., [92]) and in gravitational waves [88,93-97], to the best of our knowledge, constraints of this type have not been set before for the spacetimes considered here.

As a final remark, we note that while we have chosen only a few solutions that can be seen as deviations from the Schwarzschild/Kerr solutions since they share the same basic Einstein-Hilbert-Maxwell action of GR, the work presented here is meant largely as a proof-of-concept investigation and a methodological example of how to exploit observations and measurements that impact the photon region. While a certain degeneracy in the shadow size induced by mass and spin remains and is inevitable, when in the future the relative difference in the posterior for the angular gravitational radius for M87* can be pushed to $\lesssim 5 \%$, we should be able to constrain its spin, when modeling it as a Kerr black hole. Furthermore, since this posterior implies a spread in the estimated mass, one can expect small changes in the exact values of the maximum allowed charges reported here. Hence, as future observations-either in terms of black-hole imaging or of gravitational-wave detection-will become more precise and notwithstanding a poor measurement of the black-hole spin, the methodology presented here can be readily applied to set even tighter constraints on the physical charges of non-Einsteinian black holes.

\section{ACKNOWLEDGMENTS}

It is a pleasure to thank Enrico Barausse, Sebastian Völkel and Nicola Franchini for insightful discussions on alternative black holes. Support comes the ERC Synergy Grant "BlackHoleCam: Imaging the Event Horizon of Black Holes" (Grant No. 610058). During the completion of this work we have become aware of a related work by S. Völkel et al. [98], which deals with topics that partly overlap with those of this manuscript (i.e., EHT tests of the strong-field regime of GR). The authors of the present paper thank the following organizations and programs: the Academy of Finland (projects No. 274477, No. 284495, No. 312496, No. 315721); the Alexander von Humboldt Stiftung; Agencia Nacional de Investigación y Desarrollo (ANID), Chile via NCN19\_058 (TITANs), and Fondecyt 3190878; an Alfred P. Sloan Research Fellowship; Allegro, the European ALMA Regional Centre node in the Netherlands, the NL astronomy research network NOVA and the astronomy institutes of the University of Amsterdam, Leiden University and Radboud University; the Black Hole Initiative at Harvard University, through a grant (60477) from the John Templeton Foundation; the China Scholarship Council; Consejo Nacional de Ciencia y Tecnología (CONACYT, Mexico, projects No. U0004246083, No. U0004-259839, No. F0003-272050, No. M0037-279006, No. F0003-281692, No. 104497, No. 275201, No. 263356); the Delaney Family via the Delaney Family John A.Wheeler Chair at Perimeter Institute; Dirección General de Asuntos del Personal Académico-Universidad Nacional Autónomade México (DGAPA-UNAM, projects No. IN112417 and No. IN112820); the EACOA Fellowship of the East Asia Core Observatories Association; the European Research Council Synergy Grant "BlackHoleCam: Imaging the Event Horizon of Black Holes" (Grant No. 610058); the Generalitat Valenciana postdoctoral grant APOSTD/2018/177 and GenT Program (project No. CIDEGENT/2018/021); MICINN Research Project No. PID2019-108995GB-C22; the Gordon and Betty Moore Foundation (Grants No. GBMF-3561, 
No. GBMF-5278); the Istituto Nazionale di Fisica Nucleare (INFN) sezione di Napoli, iniziative specifiche TEONGRAV; the International Max Planck Research School for Astronomy and Astrophysics at the Universities of Bonn and Cologne; Joint Princeton/ Flatiron and Joint Columbia/Flatiron Postdoctoral Fellowships, research at the Flatiron Institute is supported by the Simons Foundation; the Japanese Government (Monbukagakusho: MEXT) Scholarship; the Japan Society for the Promotion of Science (JSPS) Grant-inAid for JSPS Research Fellowship (JP17J08829); the Key Research Program of Frontier Sciences, Chinese Academy of Sciences (CAS, grants No. QYZDJ-SSW-SLH057, No. QYZDJSSW-SYS008, No. ZDBS-LY-SLH011); the Lever-hulme Trust Early Career Research Fellowship; the Max-Planck-Gesellschaft (MPG); the Max Planck Partner Group of the MPG and the CAS; the MEXT/JSPS KAKENHI (Grants No. 18KK0090, No. JP18K13594, No. JP18K03656, No. JP18H03721, No. 18K03709, No. 18H01245, No. 25120007); the Malaysian Fundamental Research Grant Scheme (FRGS) FRGS/1/ 2019/STG02/UM/02/6; the MIT International Science and Technology Initiatives (MISTI) Funds; the Ministry of Science and Technology (MOST) of Taiwan (105-2112M-001-025-MY3, 106-2112-M-001-011， 106-2119-M001-027, 107-2119-M-001-017, 107-2119-M-001-020, 107-2119-M-110-005,108-2112-M-001-048, and 1092124-M-001-005); the National Aeronautics and Space Administration (NASA, Fermi Guest Investigator grant 80NSSC20K1567 and 80NSSC20K1567, NASA Astrophysics Theory Program Grant No. 80NSSC20K0527, NASA NuSTAR Award No. 80NSSC20K0645, NASA Grant No. NNX17AL82G, and Hubble Fellowship Grant No. HST-HF2-51431.001-A awarded by the Space Telescope Science Institute, which is operated by the Association of Universities for Research in Astronomy, Inc., for NASA, under contract NAS5-26555); the National Institute of Natural Sciences (NINS) of Japan; the National Key Research and Development Program of China (Grants No. 2016YFA0400704, No. 2016YFA0400702); the National Science Foundation (NSF, Grants No. AST0096454, No. AST-0352953, No. AST-0521233, No. AST-0705062, No. AST-0905844, No. AST0922984, No. AST-1126433, No. AST-1140030, No. DGE-1144085, No. AST-1207704, No. AST1207730, No. AST-1207752, No. MRI-1228509, No. OPP-1248097, No. AST-1310896, No. AST1337663, No. AST-1440254, No. AST-1555365, No. AST-1615796, No. AST-1715061, No. AST1716327, No. AST-1716536, No. OISE-1743747, No. AST-1816420, No. AST-1903847, No. AST1935980, No. AST-2034306); the Natural Science Foundation of China (Grants No. 11573051, No. 11633006, No. 11650110427, No. 10625314,
No. 11721303, No. 11725312, No. 11933007 , No. 11991052, No. 11991053); a fellowship of China Postdoctoral Science Foundation (2020M671266); the Natural Sciences and Engineering Research Council of Canada (NSERC, including a Discovery Grant and the NSERC Alexander Graham Bell Canada Graduate Scholarships-Doctoral Program); the National Research Foundation of Korea (the Global PhD Fellowship Grant: grants No. 2014H1A2A1018695, No. NRF2015H1A2A1033752, No. 2015-R1D1A1A01056807, the Korea Research Fellowship Program: NRF2015H1D3A1066561, Basic Research Support Grant No. 2019R1F1A1059721); the Netherlands Organization for Scientific Research (NWO) VICI award (Grant No. 639.043.513) and Spinoza Prize SPI 78-409; the New Scientific Frontiers with Precision Radio Interferometry Fellowship awarded by the South African Radio Astronomy Observatory (SARAO), which is a facility of the National Research Foundation (NRF), an agency of the Department of Science and Innovation (DSI) of South Africa; the South African Research Chairs Initiative of the Department of Science and Innovation and National Research Foundation; the Onsala Space Observatory (OSO) national infrastructure, for the provisioning of its facilities/observational support (OSO receives funding through the Swedish Research Council under Grant No. 2017-00648) the Perimeter Institute for Theoretical Physics (research at Perimeter Institute is supported by the Government of Canada through the Department of Innovation, Science and Economic Development and by the Province of Ontario through the Ministry of Research, Innovation and Science); the Spanish Ministerio de Ciencia e Innovación (grants No. PGC2018-098915-B-C21, No. AYA2016-80889-P; No. PID2019-108995GB-C21, No. PGC2018-098915-BC21); the State Agency for Research of the Spanish MCIU through the "Center of Excellence Severo Ochoa" award for the Instituto de Astrofísica de Andalucía (SEV-20170709); the Toray Science Foundation; the Consejería de Economía, Conocimiento, Empresas y Universidad, Junta de Andalucía (Grant No. P18-FR-1769), the Consejo Superior de Investigaciones Científicas (Grant No. 2019AEP112); the U.S. Department of Energy (USDOE) through the Los Alamos National Laboratory (operated by Triad National Security, LLC, for the National Nuclear Security Administration of the USDOE (Contract No. 89233218CNA000001); the European Union's Horizon 2020 research and innovation program under grant agreement No. 730562 RadioNet; ALMA North America Development Fund; the Academia Sinica; Chandra TM617006X; Chandra award DD7-18089X. This work used the Extreme Science and Engineering Discovery Environment (XSEDE), supported by NSF grant ACI-1548562, and CyVerse, supported by NSF Grants No. DBI-0735191, No. DBI-1265383, and No. DBI-1743442. XSEDE 
Stampede2 resource at TACC was allocated through TGAST170024 and TG-AST080026N. XSEDE Jet-Stream resource at PTI and TACC was allocated through AST170028. The simulations were performed in part on the SuperMUC cluster at the LRZ in Garching, on the LOEWE cluster in CSC in Frankfurt, and on the HazelHen cluster at the HLRS inStuttgart. This research was enabled in part by support provided by Compute Ontario [99], Calcul Quebec [100] and Compute Canada [101]. We thank the staff at the participating observatories, correlation centers, and institutions for their enthusiastic support. This paper makes use of the following ALMA data: ADS/JAO.ALMA\\#2016.1.01154.V. ALMA is a partnership of the European Southern Observatory (ESO; Europe, representing its member states), NSF, and National Institutes of Natural Sciences of Japan, together with National Research Council (Canada), Ministry of Science and Technology (MOST; Taiwan), Academia Sinica Institute of Astronomy and Astro-physics(ASIAA; Taiwan), and Korea Astronomy and Space Science Institute (KASI; Republic of Korea), in cooperation with the Republic of Chile. The Joint ALMA Observatory is operated by ESO, Associated Universities, Inc. (AUI)/ NRAO, and the National Astronomical Observatory of Japan (NAOJ). The NRAO is a facility of the NSF operated under cooperative agreement by AUI. This paper has made use of the following APEX data: Project ID T-091.F-00062013. APEX is a collaboration between the Max-PlanckInstitut für Radioastronomie (Germany), ESO, and the Onsala Space Observatory (Sweden). The SMA is a joint project between the SAO and ASIAA and is funded by the Smithsonian Institution and the Academia Sinica. The JCMT is operated by the East Asian Observatory on behalf of the NAOJ, ASIAA, and KASI, as well as the Ministry of Finance of China, Chinese Academy of Sciences, and the National Key R\&D Program (No. 2017YFA0402700) of China. Additional funding support for the JCMT is provided by the Science and Technologies Facility Council (UK) and participating universities in the UK and Canada. The LMT is a project operated by the Instituto Nacional de Astrofísica, Óptica y Electrónica (Mexico) and the University of Massachusetts at Amherst (USA), with financial support from the Consejo Nacional de Ciencia y Tecnología and the National Science Foundation. The IRAM 30-m telescope on Pico Veleta, Spain is operated by IRAM and supported by CNRS (Centre National de la Recherche Scientifique, France), MPG (Max-PlanckGesellschaft, Germany) and IGN (Instituto Geográfico Nacional, Spain). The SMT is operated by the Arizona Radio Observatory, a part of the Steward Observatory of the University of Arizona, with financial support of operations from the State of Arizona and financial support for instrumentation development from the NSF. The SPT is supported by the National Science Foundation through Grant No. PLR-1248097. Partial support is also provided by the NSF Physics Frontier Center Grant No. PHY1125897 to the Kavli Institute of Cosmological Physics at the University of Chicago, the Kavli Foundation and the Gordon and Betty Moore Foundation grant GBMF 947. The SPT hydrogen maser was provided on loan from the GLT, courtesy of ASIAA. The EHTC has received generous donations of FPGA chips from Xilinx Inc., under the Xilinx University Program. The EHTC has benefited from technology shared under open-source license by the Collaboration for Astronomy Signal Processing and Electronics Research (CASPER). The EHT project is grateful to T4Science and Microsemi for their assistance with Hydrogen Masers. This research has made use of NASA's Astrophysics Data System. We gratefully acknowledge the support provided by the extended staff of the ALMA, both from the inception of the ALMA Phasing Project through the observational campaigns of 2017 and 2018. We would like to thank A. Deller and W. Brisken for EHT-specific support with the use of DiFX. We acknowledge the significance that Maunakea, where the SMA and JCMT EHT stations are located, has for the indigenous Hawaiian people. Facilities: EHT, ALMA, APEX, IRAM:30 m, JCMT, LMT, SMA, ARO:SMT, SPT. Software: AIPS [102], ParselTongue [103], GNU Parallel [104], eht-imaging [105], Difmap [106], Numpy [107], Scipy [108], Pandas [109], Astropy [110,111], Jupyter [112], Matplotlib [113], THEMIS [114], DMC [115], polsolve [116], GPCAL [117].

\section{APPENDIX: DISTORTION PARAMETERS}

Since the boundary of the shadow region is a closed curve as discussed above, one can define various characteristic features for a quantitative comparison $[80,83]$. Out of the many possible measures of distortion of this curve from a perfect circle discussed in Ref. [83], we use here the simplest one which was originally introduced in Ref. [80], namely

$$
\delta_{\mathrm{sh}}=\frac{\alpha_{l, \mathrm{c}}-\alpha_{l}}{r_{\mathrm{sh}, \mathrm{c}}},
$$

where $r_{\mathrm{sh}, \mathrm{c}}$ is the radius of the circumcircle passing through the two points (since the images here are symmetric about the $\alpha$-axis) with coordinates $\left(\alpha_{\mathrm{r}}, 0\right)$ and $\left(\alpha_{\mathrm{t}}, \beta_{\mathrm{t}}\right)$, which are the rightmost and topmost points of the shadow curve, and is given as [80],

$$
r_{\mathrm{sh}, \mathrm{c}}=\frac{\left(\alpha_{\mathrm{t}}-\alpha_{\mathrm{r}}\right)^{2}+\beta_{\mathrm{t}}^{2}}{2\left|\alpha_{\mathrm{t}}-\alpha_{\mathrm{r}}\right|},
$$

with $\left(\alpha_{l}, 0\right)$ and $\left(\alpha_{l, \mathrm{c}}, 0\right)$ the leftmost points of the shadow curve and of the circumcircle respectively (see Fig. 3 of [57]).

In Fig. 4 we display the distortion parameter $\delta_{\text {sh }}$ for the shadow curves of various rotating black holes, for an equatorial observer, as an additional simple comparable characteristic. We note also that the deviation of $\delta_{\mathrm{sh}}$ from 


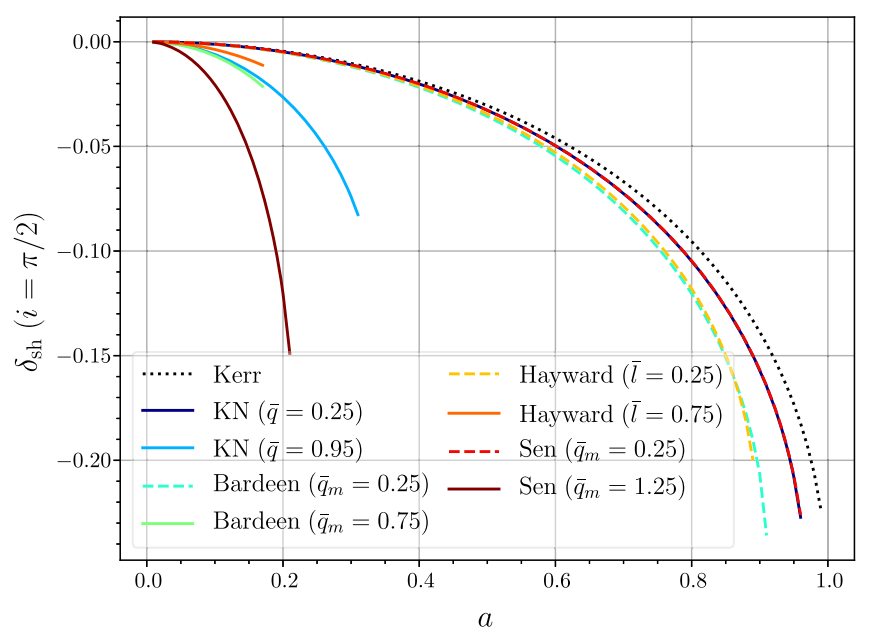

FIG. 4. Distortion parameter $\delta_{\text {sh }}$ for a number of stationary black holes observed on the equatorial plane $(i=\pi / 2)$ with dimensionless spin $a$. Because for observers viewing the black hole from inclinations increasingly close to the pole, the shadow boundary appears increasingly circular, the distortions reported can be taken as upper limits.

zero is insignificant for observer viewing angles that are close to the pole of the black hole, as anticipated (not displayed here).
As a concluding remark we note that the EHT bounds on the size of the shadow of M87*, as discussed above and displayed in Eq. (14), do not impose straightforward bounds on its shape. In particular, we can see from Fig. 4 that the rotating Bardeen black hole with $\bar{q}_{m}=$ 0.25 for high spins can be more distorted from a circle than a Kerr black hole but still be compatible with the EHT measurement (see Fig. 2). On the other hand, even though we are able to exclude Sen black holes with large electromagnetic charges (see, e.g., the Sen curve for $\bar{q}_{m}=$ 1.25 in the right panel of Fig. 2) as viable models for M87*, its shadow is less distorted from a circle than that of an extremal Kerr black hole (see Fig. 4). In other words, the examples just made highlight the importance of using the appropriate bounds on a sufficiently robust quantity when using the EHT measurement to test theories of gravity. Failing to do so may lead to incorrect bounds on the blackhole properties. For instance, Ref. [54] is able to set bounds on the parameter space of the uncharged, rotating Hayward black hole by imposing bounds on the maximum distortion of the shape of its shadow boundaries, albeit using a different measure for the distortion from a circle [see Eq. (58) there], whereas we have shown that this is not possible, upon using the bounds $4.31 M-6.08 M$ for the size of their shadows (cf. right panel of Fig. 2).
[1] R. H. Dicke, Republication of: The theoretical significance of experimental relativity, Gen. Relativ. Gravit. 51, 57 (2019).

[2] C. M. Will, The confrontation between general relativity and experiment, Living Rev. Relativity 9, 3 (2006).

[3] T. E. Collett, L. J. Oldham, R. J. Smith, M. W. Auger, K. B. Westfall, D. Bacon, R. C. Nichol, K. L. Masters, K. Koyama, and R. van den Bosch, A precise extragalactic test of General Relativity, Science 360, 1342 (2018).

[4] G. 't Hooft and M. Veltman, One-loop divergencies in the theory of gravitation, Ann. Inst. Henri Poincare Sect. A 20 , 69 (1974).

[5] N. V. Krasnikov, Nonlocal gauge theories, Theor. Math. Phys. 73, 1184 (1987).

[6] H. Lü, A. Perkins, C. N. Pope, and K. S. Stelle, Black Holes in Higher Derivative Gravity, Phys. Rev. Lett. 114, 171601 (2015).

[7] J. Scherk and J. H. Schwarz, How to get masses from extra dimensions, Nucl. Phys. B153, 61 (1979).

[8] M. B. Green, J. H. Schwarz, and E. Witten, Superstring Theory (Cambridge University Press, Cambridge, England, 1988).

[9] E. Barausse, T. Jacobson, and T. P. Sotiriou, Black holes in Einstein-aether and Hořava-Lifshitz gravity, Phys. Rev. D 83, 124043 (2011).
[10] E. Barausse and T. P. Sotiriou, Black holes in Lorentzviolating gravity theories, Classical Quantum Gravity 30, 244010 (2013).

[11] E. Barausse, T. P. Sotiriou, and I. Vega, Slowly rotating black holes in Einstein-æther theory, Phys. Rev. D 93, 044044 (2016).

[12] O. Ramos and E. Barausse, Constraints on Hořava gravity from binary black hole observations, Phys. Rev. D 99, 024034 (2019).

[13] O. Sarbach, E. Barausse, and J. A. Preciado-López, Wellposed Cauchy formulation for Einstein-æther theory, Classical Quantum Gravity 36, 165007 (2019).

[14] T. Damour and J. H. Taylor, Strong-field tests of relativistic gravity and binary pulsars, Phys. Rev. D 45, 1840 (1992).

[15] N. Wex, Testing relativistic gravity with radio pulsars, arXiv:1402.5594.

[16] N. Wex and M. Kramer, Gravity tests with radio pulsars, Universe 6, 156 (2020).

[17] R. Abuter et al. (GRAVITY Collaboration), Detection of the gravitational redshift in the orbit of the star S2 near the Galactic centre massive black hole, Astron. Astrophys. 615, L15 (2018).

[18] R. Abuter, A. Amorim, M. Bauböck, J. P. Berger, H. Bonnet, W. Brandner, V. Cardoso, Y. Clénet, P. T. de Zeeuw, and J. Dexter, Detection of the Schwarzschild precession in the orbit of the star S2 near the Galactic 
centre massive black hole, Astron. Astrophys. 636, L5 (2020).

[19] B. P. Abbott, R. Abbott, T. D. Abbott, M. R. Abernathy, F. Acernese, K. Ackley, C. Adams, T. Adams, P. Addesso, R. X. Adhikari et al., Observation of Gravitational Waves from a Binary Black Hole Merger, Phys. Rev. Lett. 116, 061102 (2016).

[20] B. P. Abbott, R. Abbott, T. D. Abbott, M. R. Abernathy, F. Acernese, K. Ackley, C. Adams, T. Adams, P. Addesso, R. X. Adhikari et al., Astrophysical implications of the binary black hole merger GW150914, Astrophys. J. Lett. 818, L22 (2016).

[21] K. Akiyama, A. Alberdi, W. Alef, K. Asada, R. Azulay, A.-K. Baczko, D. Ball, M. Baloković, J. Barrett et al. (Event Horizon Telescope Collaboration), First M87 event horizon telescope results. I. The shadow of the supermassive black hole, Astrophys. J. Lett. 875, L1 (2019).

[22] K. Akiyama, A. Alberdi, W. Alef, K. Asada, R. Azulay, A.-K. Baczko, D. Ball, M. Baloković, J. Barrett et al. (Event Horizon Telescope Collaboration), First M87 event horizon telescope results. II. Array and instrumentation, Astrophys. J. Lett. 875, L2 (2019).

[23] K. Akiyama, A. Alberdi, W. Alef, K. Asada, R. Azulay, A.-K. Baczko, D. Ball, M. Baloković, J. Barrett et al. (Event Horizon Telescope Collaboration), First M87 event horizon telescope results. III. Data processing and calibration, Astrophys. J. Lett. 875, L3 (2019).

[24] K. Akiyama, A. Alberdi, W. Alef, K. Asada, R. Azulay, A.-K. Baczko, D. Ball, M. Baloković, J. Barrett et al. (Event Horizon Telescope Collaboration), First M87 event horizon telescope results. IV. Imaging the central supermassive black hole, Astrophys. J. Lett. 875, L4 (2019).

[25] K. Akiyama, A. Alberdi, W. Alef, K. Asada, R. Azulay, A.-K. Baczko, D. Ball, M. Baloković, J. Barrett et al. (Event Horizon Telescope Collaboration), First M87 event horizon telescope results. V. Physical origin of the asymmetric ring, Astrophys. J. Lett. 875, L5 (2019).

[26] K. Akiyama, A. Alberdi, W. Alef, K. Asada, R. Azulay, A.-K. Baczko, D. Ball, M. Baloković, J. Barrett et al. (Event Horizon Telescope Collaboration), First M87 event horizon telescope results. VI. The shadow and mass of the central black hole, Astrophys. J. Lett. 875, L6 (2019).

[27] K. Gebhardt, J. Adams, D. Richstone, T. R. Lauer, S. M. Faber, K. Gültekin, J. Murphy, and S. Tremaine, The black hole mass iN M87 from GEMINI/NIFS adaptive optics observations, Astrophys. J. 729, 119 (2011).

[28] D. Psaltis, L. Medeiros, P. Christian, F. Ozel, and the EHT Collaboration, Gravitational Test beyond the First PostNewtonian Order with the Shadow of the M87 Black Hole, Phys. Rev. Lett. 125, 141104 (2020).

[29] T. Johannsen and D. Psaltis, Metric for rapidly spinning black holes suitable for strong-field tests of the no-hair theorem, Phys. Rev. D 83, 124015 (2011).

[30] T. Johannsen, Systematic study of event horizons and pathologies of parametrically deformed Kerr spacetimes, Phys. Rev. D 87, 124017 (2013).

[31] S. Vigeland, N. Yunes, and L. C. Stein, Bumpy black holes in alternative theories of gravity, Phys. Rev. D 83, 104027 (2011).
[32] T. Johannsen, Regular black hole metric with three constants of motion, Phys. Rev. D 88, 044002 (2013).

[33] L. Rezzolla and A. Zhidenko, New parametrization for spherically symmetric black holes in metric theories of gravity, Phys. Rev. D 90, 084009 (2014).

[34] Z. Younsi, A. Zhidenko, L. Rezzolla, R. Konoplya, and Y. Mizuno, New method for shadow calculations: Application to parametrized axisymmetric black holes, Phys. Rev. D 94, 084025 (2016).

[35] R. Konoplya, L. Rezzolla, and A. Zhidenko, General parametrization of axisymmetric black holes in metric theories of gravity, Phys. Rev. D 93, 064015 (2016).

[36] P. Kocherlakota and L. Rezzolla, Accurate mapping of spherically symmetric black holes in a parametrized framework, Phys. Rev. D 102, 064058 (2020).

[37] C. Bambi and K. Freese, Apparent shape of super-spinning black holes, Phys. Rev. D 79, 043002 (2009).

[38] C. Bambi and N. Yoshida, Shape and position of the shadow in the $\delta=2$ Tomimatsu-Sato spacetime, Classical Quantum Gravity 27, 205006 (2010).

[39] L. Amarilla, E. F. Eiroa, and G. Giribet, Null geodesics and shadow of a rotating black hole in extended Chern-Simons modified gravity, Phys. Rev. D 81, 124045 (2010).

[40] L. Amarilla and E. F. Eiroa, Shadow of a Kaluza-Klein rotating dilaton black hole, Phys. Rev. D 87, 044057 (2013).

[41] S.-W. Wei and Y.-X. Liu, Observing the shadow of Einstein-Maxwell-Dilaton-Axion black hole, J. Cosmol. Astropart. Phys. 11 (2013) 063.

[42] P. G. Nedkova, V. K. Tinchev, and S. S. Yazadjiev, Shadow of a rotating traversable wormhole, Phys. Rev. D 88, 124019 (2013).

[43] U. Papnoi, F. Atamurotov, S. G. Ghosh, and B. Ahmedov, Shadow of five-dimensional rotating Myers-Perry black hole, Phys. Rev. D 90, 024073 (2014).

[44] S.-W. Wei, P. Cheng, Y. Zhong, and X.-N. Zhou, Shadow of noncommutative geometry inspired black hole, J. Cosmol. Astropart. Phys. 08 (2015) 004.

[45] M. Ghasemi-Nodehi, Z. Li, and C. Bambi, Shadows of CPR black holes and tests of the Kerr metric, Eur. Phys. J. C 75, 315 (2015).

[46] F. Atamurotov, S. G. Ghosh, and B. Ahmedov, Horizon structure of rotating Einstein-Born-Infeld black holes and shadow, Eur. Phys. J. C 76, 273 (2016).

[47] B. P. Singh and S. G. Ghosh, Shadow of SchwarzschildTangherlini black holes, arXiv:1707.07125.

[48] M. Amir, B. P. Singh, and S. G. Ghosh, Shadows of rotating five-dimensional charged EMCS black holes, Eur. Phys. J. C 78, 399 (2018).

[49] H. Olivares, Z. Younsi, C. M. Fromm, M. De Laurentis, O. Porth, Y. Mizuno, H. Falcke, M. Kramer, and L. Rezzolla, How to tell an accreting boson star from a black hole, Mon. Not. R. Astron. Soc. 497, 521 (2020).

[50] Y. Mizuno, Z. Younsi, C. M. Fromm, O. Porth, M. De Laurentis, H. Olivares, H. Falcke, M. Kramer, and L. Rezzolla, The current ability to test theories of gravity with black hole shadows, Nat. Astron. 2, 585 (2018).

[51] P. V. P. Cunha, C. A. R. Herdeiro, and E. Radu, Spontaneously Scalarized Kerr Black Holes in Extended 
Scalar-Tensor-Gauss-Bonnet Gravity, Phys. Rev. Lett. 123, 011101 (2019).

[52] A. Grenzebach, The Shadow of Black Holes (Springer International Publishing, New York, 2016).

[53] Z. Stuchlík and J. Schee, Shadow of the regular Bardeen black holes and comparison of the motion of photons and neutrinos, Eur. Phys. J. C 79, 44 (2019).

[54] R. Kumar, S. G. Ghosh, and A. Wang, Shadow cast and deflection of light by charged rotating regular black holes, Phys. Rev. D 100, 124024 (2019).

[55] R. Kumar, A. Kumar, and S. G. Ghosh, Testing rotating regular metrics as candidates for astrophysical black holes, Astrophys. J. 896, 89 (2020).

[56] K. Hioki and U. Miyamoto, Hidden symmetries, null geodesics, and photon capture in the Sen black hole, Phys. Rev. D 78, 044007 (2008).

[57] A. Abdujabbarov, M. Amir, B. Ahmedov, and S. G. Ghosh, Shadow of rotating regular black holes, Phys. Rev. D 93, 104004 (2016).

[58] P. O. Mazur and E. Mottola, Gravitational vacuum condensate stars, Proc. Natl. Acad. Sci. U.S.A. 101, 9545 (2004).

[59] C. B. M. H. Chirenti and L. Rezzolla, Ergoregion instability in rotating gravastars, Phys. Rev. D 78, 084011 (2008).

[60] R. Shaikh, P. Kocherlakota, R. Narayan, and P. S. Joshi, Shadows of spherically symmetric black holes and naked singularities, Mon. Not. R. Astron. Soc. 482, 52 (2019).

[61] D. Dey, R. Shaikh, and P. S. Joshi, Shadow of nullike and timelike naked singularities without photon spheres, Phys. Rev. D 103, 024015 (2021).

[62] R. M. Wald, General Relativity (The University of Chicago Press, Chicago, 1984).

[63] J. Bardeen, in Proceedings of GR5, Tbilisi, USSR (Georgia, 1968), p. 174.

[64] S. A. Hayward, Formation and Evaporation of Nonsingular Black Holes, Phys. Rev. Lett. 96, 031103 (2006).

[65] V. P. Frolov, Notes on nonsingular models of black holes, Phys. Rev. D 94, 104056 (2016).

[66] D. I. Kazakov and S. N. Solodukhin, On quantum deformation of the Schwarzschild solution, Nucl. Phys. B429, 153 (1994).

[67] G. W. Gibbons and K.-I. Maeda, Black holes and membranes in higher-dimensional theories with dilaton fields, Nucl. Phys. B298, 741 (1988).

[68] D. Garfinkle, G. T. Horowitz, and A. Strominger, Charged black holes in string theory, Phys. Rev. D 43, 3140 (1991).

[69] author A. García, D. Galtsov, and O. Kechkin, Class of Stationary Axisymmetric Solutions of the Einstein-Maxwell-Dilaton-Axion Field Equations, Phys. Rev. Lett. 74, 1276 (1995).

[70] R. Kallosh, A. Linde, T. Ortín, A. Peet, and A. van Proeyen, Supersymmetry as a cosmic censor, Phys. Rev. D 46, 5278 (1992).

[71] A. I. Janis, E. T. Newman, and J. Winicour, Reality of the Schwarzschild Singularity, Phys. Rev. Lett. 20, 878 (1968).

[72] R. P. Kerr, Gravitational Field of a Spinning Mass as an Example of Algebraically Special Metrics, Phys. Rev. Lett. 11, 237 (1963).
[73] E. T. Newman, E. Couch, K. Chinnapared, A. Exton, A. Prakash, and R. Torrence, Metric of a rotating, charged mass, J. Math. Phys. (N.Y.) 6, 918 (1965).

[74] A. Sen, Rotating charged black hole solution in heterotic string theory, Phys. Rev. Lett. 69, 1006 (1992).

[75] C. Bambi and L. Modesto, Rotating regular black holes, Phys. Lett. B 721, 329 (2013).

[76] E. T. Newman and A. I. Janis, Note on the Kerr spinningparticle metric, J. Math. Phys. (N.Y.) 6, 915 (1965).

[77] B. Carter, Global structure of the Kerr family of gravitational fields, Phys. Rev. 174, 1559 (1968).

[78] F. Astorga, J. F. Salazar, and T. Zannias, On the integrability of the geodesic flow on a Friedmann-RobertsonWalker spacetime, Phys. Scr. 93, 085205 (2018).

[79] S. Yazadjiev, LETTER: Newman-Janis method and rotating dilaton-axion black hole, Gen. Relativ. Gravit. 32, 2345 (2000).

[80] K. Hioki and K.-I. Maeda, Measurement of the Kerr spin parameter by observation of a compact object's shadow, Phys. Rev. D 80, 024042 (2009).

[81] R. Shaikh, Black hole shadow in a general rotating spacetime obtained through Newman-Janis algorithm, Phys. Rev. D 100, 024028 (2019).

[82] J. M. Bardeen, W. H. Press, and S. A. Teukolsky, Rotating black holes: Locally nonrotating frames, energy extraction, and scalar synchrotron radiation, Astrophys. J. 178, 347 (1972).

[83] A. A. Abdujabbarov, L. Rezzolla, and B. J. Ahmedov, A coordinate-independent characterization of a black hole shadow, Mon. Not. R. Astron. Soc. 454, 2423 (2015).

[84] T. Johannsen and D. Psaltis, Testing the no-hair theorem with observations in the electromagnetic spectrum. II. Black hole images, Astrophys. J. 718, 446 (2010).

[85] M. E. Rodrigues and M. V.d. S. Silva, Bardeen regular black hole with an electric source, J. Cosmol. Astropart. Phys. 06 (2018) 025.

[86] S. L. Liebling and C. Palenzuela, Dynamical boson stars, Living Rev. Relativity 15, 6 (2012).

[87] Z. Meliani, P. Grandclément, F. Casse, F. H. Vincent, O. Straub, and F. Dauvergne, GR-AMRVAC code applications: accretion onto compact objects, boson stars versus black holes, Classical Quantum Gravity 33, 155010 (2016).

[88] E. W. Hirschmann, L. Lehner, S. L. Liebling, and C. Palenzuela, Black hole dynamics in Einstein-Maxwelldilaton theory, Phys. Rev. D 97, 064032 (2018).

[89] J. Magueijo, New varying speed of light theories, Rep. Prog. Phys. 66, 2025 (2003).

[90] D. L. Wiltshire, M. Visser, and S. M. Scott, The Kerr Spacetime: Rotating Black Holes in General Relativity (Cambridge University Press, Cambridge, England, 2009).

[91] A. Held, R. Gold, and A. Eichhorn, Asymptotic safety casts its shadow, J. Cosmol. Astropart. Phys. 06 (2019) 029.

[92] C. Bambi, Testing the Bardeen metric with the black hole candidate in Cygnus X-1, Phys. Lett. B 730, 59 (2014).

[93] E. Barausse, N. Yunes, and K. Chamberlain, TheoryAgnostic Constraints on Black-Hole Dipole Radiation with Multiband Gravitational-Wave Astrophysics, Phys. Rev. Lett. 116, 241104 (2016). 
[94] R. Konoplya and A. Zhidenko, Detection of gravitational waves from black holes: Is there a window for alternative theories? Phys. Lett. B 756, 350 (2016).

[95] F.-L. Julié, On the motion of hairy black holes in EinsteinMaxwell-dilaton theories, J. Cosmol. Astropart. Phys. 01 (2018) 026.

[96] F.-L. Julié, Gravitational radiation from compact binary systems in Einstein-Maxwell-dilaton theories, J. Cosmol. Astropart. Phys. 10 (2018) 033.

[97] H. M. Siahaan, Merger estimates for Kerr-Sen black holes, Phys. Rev. D 101, 064036 (2020).

[98] S. H. Völkel, E. Barausse, N. Franchini, and A. E. Broderick, EHT tests of the strong-field regime of General Relativity, arXiv:2011.06812.

[99] http://computeontario.ca.

[100] http://www.calculquebec.ca.

[101] http://www.computecanada.ca.

[102] E. W. Greisen, AIPS, the VLA, and the VLBA, in In: Heck A. (eds) Information Handling in Astronomy - Historical Vistas, edited by A. Heck, Astrophysics and Space Science Library Vol. 285 (Springer, Dordrecht, 2003), https://doi .org/10.1007/0-306-48080-8_7.

[103] M. Kettenis, H. J. van Langevelde, C. Reynolds, and B. Cotton, ParselTongue: AIPS Talking Python, in Astronomical Data Analysis Software and Systems XV, edited by C. Gabriel, C. Arviset, D. Ponz, and S. Enrique, Astronomical Society of the Pacific Conference Series Vol. 351 (2006), https://ui.adsabs.harvard.edu/abs/2006ASPC..351. .497K.

[104] O. Tange, login: The USENIX Magazine 36, 42 (2011).

[105] A. A. Chael, M. D. Johnson, R. Narayan, S. S. Doeleman, J. F. C. Wardle, and K. L. Bouman, High-resolution linear polarimetric imaging for the event horizon telescope, Astrophys. J. 829, 11 (2016).
[106] M. Shepherd, https://ui.adsabs.harvard.edu/abs/2011ascl .soft03001S.

[107] S. van der Walt, S. C. Colbert, and G. Varoquaux, The NumPy array: A structure for efficient numerical computation, Comput. Sci. Eng. 13, 22 (2011).

[108] E. Jones et al., SciPy: Open Source Scientific Tools for Python (2001), http://www.scipy.org/.

[109] W. McKinney, Proc. IX Python in Science Conf., edited by S. van der Walt and J. Millman (2010).

[110] T. P. Robitaille et al., Astropy: A community Python package for astronomy, Astron. Astrophys. 558, A33 (2013).

[111] A. M. Price-Whelan (The Astropy Collaboration), The astropy project: Building an open-science project and status of the v2.0 core package, Astron. J. 156, 123 (2018).

[112] T. Kluyver et al., Positioning and Power in Academic Publishing: Players, Agents and Agendas, edited by F. Loizides and B. Schmidt (IOS Press, 2016).

[113] J. D. Hunter, Matplotlib: A 2D graphics environment, Comput. Sci. Eng. 9, 90 (2007).

[114] A. E. Broderick, THEMIS: A parameter estimation framework for the event horizon telescope, Astrophys. J. 897, 139 (2020).

[115] D. W. Pesce, A D-term modeling code (DMC) for simultaneous calibration and full-stokes imaging of very long baseline interferometric data, Astron. J. 161, 178 (2021).

[116] I. Martí-Vidal, A. Mus, M. Janssen, P. de Vicente, and J. González, Polarization calibration techniques for the new-generation VLBI, Astron. Astrophys. 646, A52 (2021).

[117] J. Park, D.-Y. Byun, K. Asada, and Y. Yun, GPCAL: A generalized calibration pipeline for instrumental polarization in VLBI data, Astrophys. J. 906, 85 (2021). 